\title{
Miniature Dual Axes Confocal Microscope for Real Time In Vivo Imaging
}

\author{
Wibool Piyawattanametha and Thomas D. Wang \\ ${ }^{1}$ Departmentsof Applied Physics, Biology, Electrical Engineering Microbiology $\mathcal{E}$ \\ Immunology, Radiology, and Pediatrics James H. Clark Center (Bio-X), Stanford, \\ ${ }^{2}$ NECTEC, Pathumthani, \\ ${ }^{3}$ Faculty of Medicine, Chulalongkorn University, Prathumwan, \\ ${ }^{1} \mathrm{CA}$ \\ 2,3Thailand
}

\section{Introduction}

Today, disease interpretation of excised tissue is performed by analyzing biopsy specimens with a tabletop microscope [1]. While this method is effective, the process can be limited by sampling error, processing costs, and preparation time. In addition, the interpretive accuracy of the specimens can be affected by artefacts associated with tissue sectioning, paraffin embedding, and histochemical staining. Thus, a lot of effort has gone into the development of new methods that perform real time in vivo imaging with sub-cellular resolution.

Confocal microscopy is a powerful optical imaging method that can achieve sub-cellular resolution in real time. The technique of optical sectioning provides clear images from "optically thick" biological tissues that have previously been collected with large, tabletop instruments that occupy the size of a table [2,3]. They can be used to collect either reflectance or fluorescence images to identify morphological or

molecular features of cells and tissues, respectively. Moreover, images in both modalities can be captured simultaneously with complete spatial registration. This approach uses a "pinhole" placed in between the objective lens and the detector to allow only the light that originates from within a tiny focal volume below the tissue surface to be collected. For miniature instruments, the core of an optical fiber is used as the "pinhole."

Recently, significant progress has been made in the development of endoscope-compatible confocal imaging instruments for visualizing inside the human body. This direction has been accelerated by the availability, variety and low cost of optical fibers, scanners, and light sources, in particular, semiconductor lasers. These methods are being developed for use in the clinic as well as in small animal imaging facilities. The addition of a miniature real-time, high resolution imaging instrument can help guide tissue biopsy and reduce pathology costs. However, these efforts are technically challenging because of the demanding performance requirements for small instrument size, high image resolution, deep tissue penetration depths, and fast frame rates.

The performance parameters for miniature in vivo confocal imaging instruments are governed by the specific application. An important goal is the early detection and image

Source: Advances in Solid State Circuits Technologies, Book edited by: Paul K. Chu,

ISBN 978-953-307-086-5, pp. 446, April 2010, INTECH, Croatia, downloaded from SCIYO.COM 
guided therapy of disease in hollow organs, including colon, esophagus, lung, oropharynx, and cervix. Applications can also be found for better understanding of the molecular mechanisms of disease in small animals. In particular, localization of pre-malignant (dysplastic) lesions in the digestive tract can guide tissue biopsy for early detection and prevention of cancer. In addition, visualization of over expressed molecular targets in small animal models can lead to the discovery of new drugs.

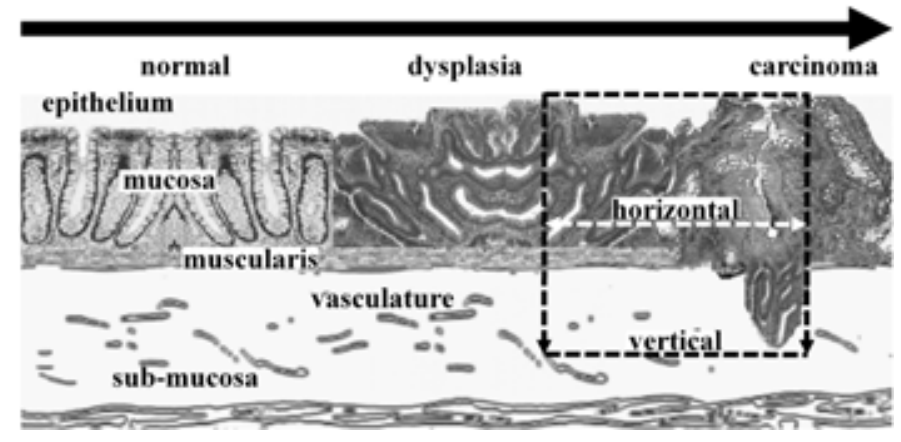

Fig. 1. Dysplasia represents a pre-malignant condition in the epithelium of hollow organs, such as the colon and esophagus. The dual axes confocal architecture has high dynamic range that is suitable for imaging in the vertical cross-sectional plane to visualize disease processes with greater tissue penetration depths.

As shown in Fig. 1, dysplasia originates in the epithelium and represents an important step in the transformation of normal mucosa to carcinoma. Dysplasia has a latency period of approximately 7 to 14 years before progressing onto cancer and offers a window of opportunity for evaluating patients by endoscopy who are at increased risk for developing cancer. The early detection and localization of dysplastic lesions can guide tissue resection and prevent future cancer progression. Dysplastic glands can be present from the mucosal surface down to the muscularis. Thus, an imaging depth of $\sim 500 \mu \mathrm{m}$ is sufficient to evaluate most early epithelial disease processes.

On reflectance imaging, sub-cellular resolution (typically $<5 \mu \mathrm{m}$ ) is needed to identify nuclear features, such as nuclear-to-cytoplasm ratio. On fluorescence imaging, high contrast is needed to distinguish between the target and background. With both modalities, a fast imaging frame rate $(>4 \mathrm{~Hz})$ is necessary to avoid motion artefact.

\section{Single axls confocal architecture}

\section{A. Configuration of optics}

Recent advances in the development of microlenses and miniature scanners have resulted in the development of fiber optic coupled instruments that are endoscope compatible with high resolution, including single [4-8], and multiple fiber [7, 9] strategies. Different methods of scanning are also being explored [10-14].

All of these endoscope compatible designs use a single axis design, where the pinhole (fiber) and objective are located along one main optical axis. A high NA objective is used to achieve sub-cellular resolution and maximum light collection, and the same objective is used for both the illumination and collection of light. In order to scale down the dimension of these 
instruments for endoscope compatibility, the diameter of the objective must be reduced to $\sim 5 \mathrm{~mm}$ or less. As a consequence, the working distance (WD) as well as the field-of-view (FOV) is also decreased, as shown by the progression of the 3 different objectives in Fig. 2. The tissue penetration depth also decreases, and is typically inadequate to assess the tissue down to the muscularis, which is located at a depth of $\sim 500 \mu \mathrm{m}$ and is an important landmark for defining the early presence of epithelial cancers.

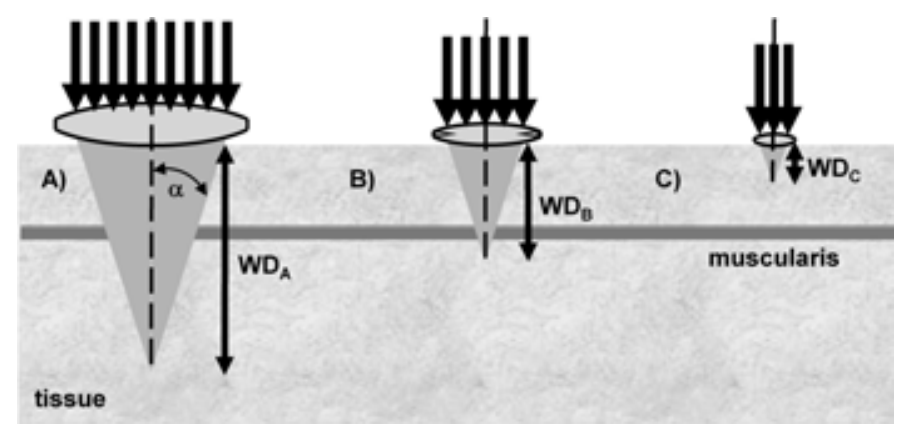

Fig. 2. For endoscope compatibility, the diameter of a single axis confocal microscope must be scaled down in size $(A \rightarrow B \rightarrow C)$, resulting in a reduced working distance and limited tissue penetration depth.

\section{B. Resolutions}

For the conventional single axis architecture, the transverse, $\Delta \mathrm{r}_{\mathrm{s}}$, and axial, $\Delta \mathrm{z}_{\mathrm{s}}$, resolution between full-width-half-power (FWHP) points for uniform illumination of the lenses are defined by the following equations [3]:

$$
\Delta r_{s}=\frac{0.37 \lambda}{n \sin \alpha} \approx \frac{0.37 \lambda}{n \alpha} ; \Delta z_{s}=\frac{0.89 \lambda}{n(1-\cos \alpha)} \approx \frac{1.78 \lambda}{n \alpha^{2}}
$$

where $\lambda$ is the wavelength, $\mathrm{n}$ is the refractive index of the medium, $\mathrm{a}$ is the maximum convergence half-angle of the beam, $N A=n \sin \alpha$ is the numerical aperture, and $\sin \alpha \approx \alpha$ for low NA lenses. Eq. (1) implies that the transverse and axial resolution varies as 1/NA and $1 / \mathrm{NA}^{2}$, respectively. A resolution of less than $5 \mu \mathrm{m}$ is adequate to identify sub-cellular structures that are important for medical and biological applications. To achieve this resolution in the axial dimension, the objective lens used requires a relatively large NA $(>0.4)$. The optics can be reduced to the millimeter scale for in vivo imaging, but requires a sacrifice of resolution, FOV, or WD. Also, a high NA objective limits the available WD, and requires that the scanning mechanism be located in the pre-objective position, restricting the FOV and further increasing sensitivity to off-axis aberrations.

\section{Commercial systems}

Two endoscope compatible confocal imaging systems are commercially available for clinical use. The EC-3870K (Pentax Precision Instruments, Tokyo, Japan) has an integrated design where a confocal module (Optiscan Pty Ltd, Victoria, Australia) is built into the insertion tube of the endoscope, and results in an overall diameter of $12.8 \mathrm{~mm}$, as shown in Fig. 3a [15]. This module uses the single axis optical configuration where a single mode optical fiber is aligned on-axis with an objective that has an NA $\approx 0.6$. Scanning of the distal tip of the 
optical fiber is performed mechanically by coupling the fiber to a tuning fork that vibrates at resonance. Axial scanning is performed with a shape memory alloy (nitinol) actuator that can translate the focal volume over a distance of 0 to $250 \mu \mathrm{m}$ below the tissue surface. Excitation is provide at $488 \mathrm{~nm}$ (peak absorption of fluorescein) by a semi-conductor laser, and a transverse and axial resolution of 0.7 and $7 \mu \mathrm{m}$, respectively, has been achieved. The images are collected at a frame rate of either 0.8 or $1.6 \mathrm{~Hz}$ to achieve a FOV of either $1024 \times 1024$ or $1024 \times 512$ pixels, respectively. The dimension of the confocal instrument by itself is $\sim 5 \mathrm{~mm}$. When a suspicious lesion is identified, the confocal window located on the distial tip is placed into contact with the tissue to collect images. A separate instrument channel can be used to obtain pinch biopsies of tissue.
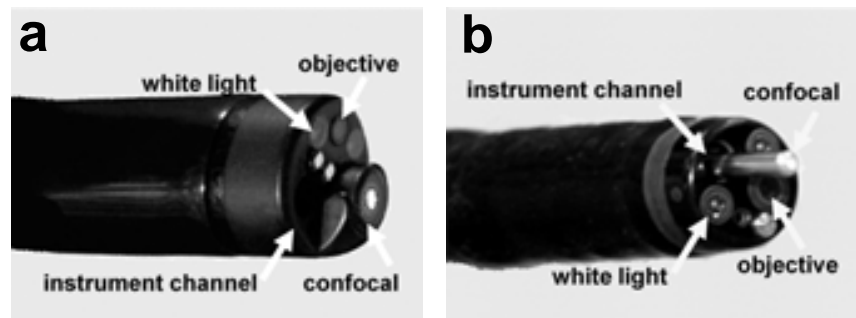

Fig. 3. a) The EC-3870K (Pentax) has a confocal module (Optiscan) integrated into the endoscope insertion tube. b) The Cellvizio ${ }^{\circledR} \mathrm{GI}$ is a confocal miniprobe that passes through the instrument channel of the endoscope.

The Cellvizio ${ }^{\circledR}$ GI (Mauna Kea Technologies, Paris, France) uses a set of miniprobes that range in diameter from 1.5 to $2.5 \mathrm{~mm}$, and passes through the standard instrument channel of medical endoscopes, as shown in Fig. 3b. This instrument moves independently of the endoscope, and its placement onto the tissue surface can be guided by the conventional white light image $[8,15]$. This miniprobe consists of a fiber bundle with $\sim 30,000$ individual fibers that is aligned on-axis with an objective that has an NA $\approx 0.6$. The core of each individual fiber acts as a collection pinhole to reject out-of-focus light. Scanning is performed at the proximal end of the bundle in the instrument control unit with a $4 \mathrm{kHz}$ oscillating mirror for horizontal lines and a $12 \mathrm{~Hz}$ galvo mirror for frames. In this design, axial scanning cannot be performed. Instead, separate miniprobes that have different working distances are needed to optically section at different depths. Excitation is provided at $488 \mathrm{~nm}$, and the transverse and axial resolution of these instruments ranges from 2.5 to 5 $\mu \mathrm{m}$ and 15 to $20 \mu \mathrm{m}$, respectively. Images are collected at a frame rate of $12 \mathrm{~Hz}$ with a FOV of either $600 \times 500 \mu \mathrm{m}^{2}$ or $240 \times 200 \mu \mathrm{m}^{2}$.

\section{Dual axes confocal architecture}

\section{Configuration of optics}

So far, the aforementioned miniaturization techniques in the previous section deploy a conventional single-axis confocal architecture that has the objective and optical fiber aligned along the same optical axis. In order to overcome some of these limitations for endoscope compatibility and in vivo imaging, we have developed the novel dual axes confocal configuration, shown in Fig. 4. We use two fibers oriented along separate optical axis of 
different low NA objectives to spatially separate the light paths for illumination and collection [16, 17]. The region of overlap between the two beams (crossed at a half angle $\theta$ from the midline) defines the focal volume, hence the resolution, and can achieve subcellular dimensions. A very low probability exists for light scattered by tissue along the illumination path (blue cone) to enter the low NA collection objective (green cone), thus significant improvement in the dynamic range of detection can be achieved.

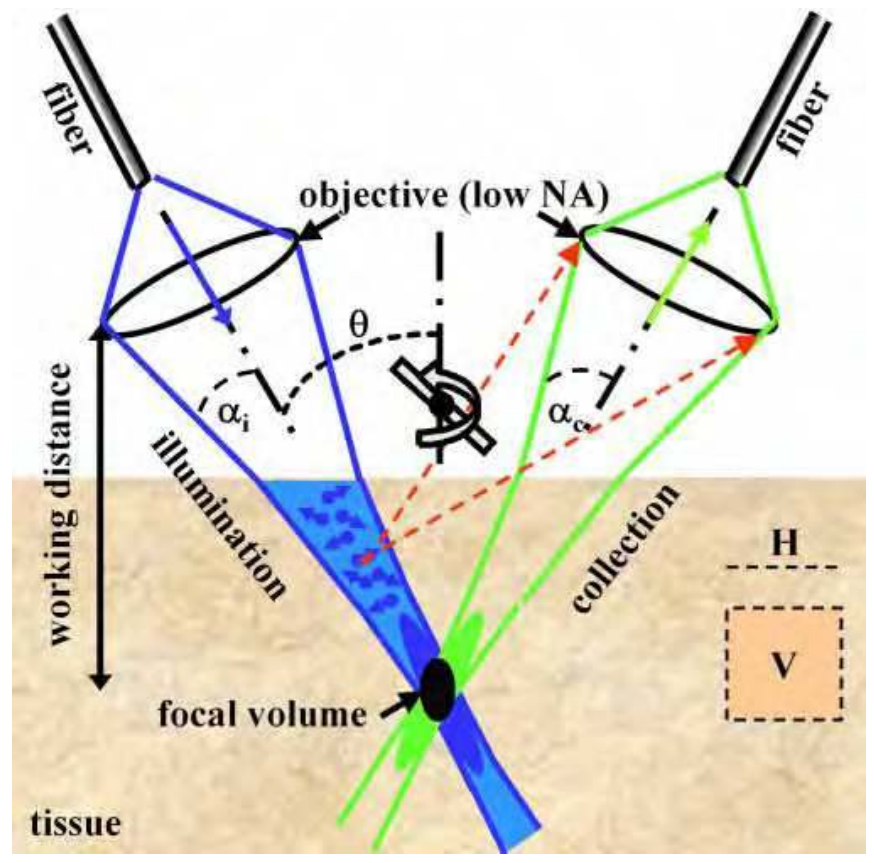

Fig. 4. Novel dual axes confocal architecture uses separate optical fibers and low NA lenses for off-axis light collection, achieving long working distance, high dynamic range, and scalability while preserving resolution.

Furthermore, the low NA objectives enable an increased working distance so that the scanning mirror can be placed on the distal (tissue) side of the lens (post-objective position), resulting in less sensitivity to off-axis aberrations [17]. In this configuration, the beams always pass through the low NA objectives on axis, resulting in a diffraction-limited focal volume that can then be scanned over a large FOV, limited by the performance of the scanner rather than by the optics. This design feature allows for the instrument to be scaled down in size to millimeter dimensions for compatibility with medical endoscopes without loss of performance.

We first develop the theory to explain the unique performance features of the dual axes confocal architecture by characterizing the point-spread function (PSF) and dynamic range. Then, we demonstrate the scaled down implementation of this configuration in miniature prototypes. Because of the challenges of packaging in such a small form factor, we first demonstrate a handheld (10 mm diameter) instrument and then an endoscope-compatible (5.5 mm diameter) prototype, using the same MEMS mirror and scanhead optics. 


\section{E. Definition of coordinates}

The coordinates for the dual axes confocal configuration are shown in Fig. 5. The illumination $(\mathrm{IO})$ and collection (CO) objectives represent separate low NA lenses. The maximum convergence half-angles of the illumination and collection beams are represented by $a_{i}$ and $a_{c}$, respectively. The separate optical axes are defined to cross the $z$-axis $\left(z_{d}\right)$ at an angle $\theta$. The main lobe of the PSF of the illumination objective is represented by the light gray oval. This lobe has a narrow transverse but a wide axial dimension.

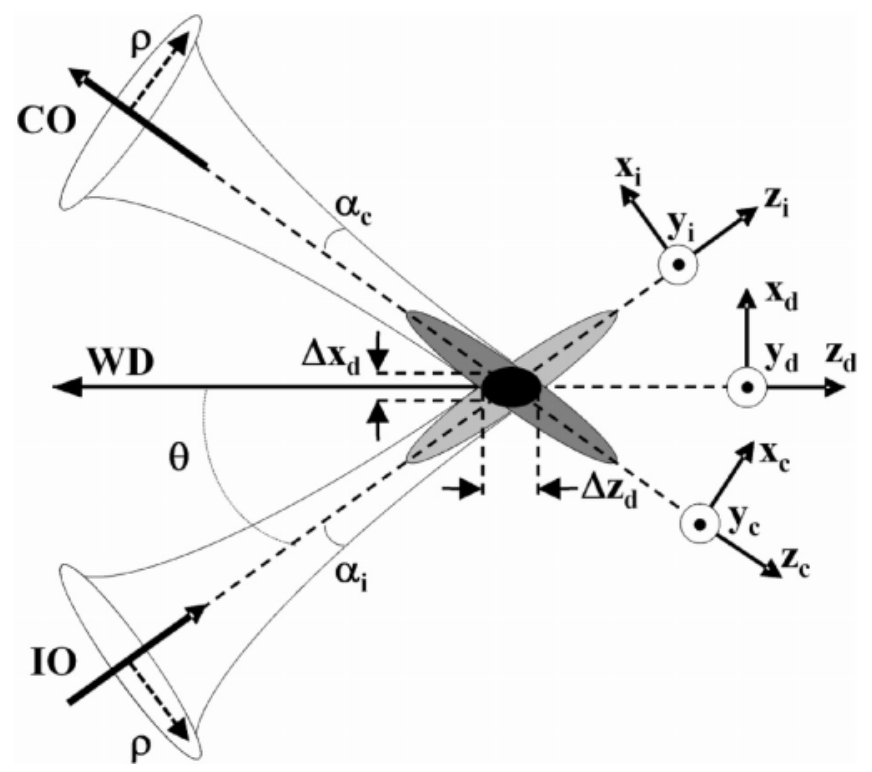

Fig. 5. Coordinates for dual axes confocal configuration

Similarly, the main lobe of the PSF of the collection objective is similar in shape but symmetrically reflected about $\mathrm{z}_{\mathrm{d}}$, as represented by the dark gray oval. For dual axes, the combined PSF is represented by the overlap of the two individual PSF's, represented by the black oval. This region is characterized by narrow transverse dimensions, $\Delta \mathrm{x}_{\mathrm{d}}$ and $\Delta \mathrm{y}_{\mathrm{d}}$ (out of the page), and by a significantly reduced axial dimension, $\Delta \mathrm{z}_{\mathrm{d}}$, which depends on the transverse rather than the axial dimension of the individual beams where they intersect.

\section{F. Point spread function}

The dual axes PSF can be derived using diffraction theory with paraxial approximations [18]. The coordinates for the illumination $\left(x_{i}, y_{i}, z_{i}\right)$ and collection $\left(x_{c}, y_{c}, z_{c}\right)$ beams are defined in terms of the coordinates of the main optical axis $\left(\mathrm{x}_{\mathrm{d}}, \mathrm{y}_{\mathrm{d}}, \mathrm{z}_{\mathrm{d}}\right)$, and may be expressed as follows:

$$
\begin{array}{ll}
\mathrm{x}_{\mathrm{i}}=\mathrm{x}_{\mathrm{d}} \cos \theta-\mathrm{z}_{\mathrm{d}} \sin \theta & \mathrm{x}_{\mathrm{c}}=\mathrm{x}_{\mathrm{d}} \cos \theta+\mathrm{z}_{\mathrm{d}} \sin \theta \\
\mathrm{y}_{\mathrm{i}}=\mathrm{y}_{\mathrm{d}} & \mathrm{y}_{\mathrm{c}}=\mathrm{y}_{\mathrm{d}} \\
\mathrm{z}_{\mathrm{i}}=\mathrm{x}_{\mathrm{d}} \sin \theta+\mathrm{z}_{\mathrm{d}} \cos \theta & \mathrm{z}_{\mathrm{c}}=-\mathrm{x}_{\mathrm{d}} \sin \theta+\mathrm{z}_{\mathrm{d}} \cos \theta
\end{array}
$$

The maximum convergence half-angles of the focused illumination and collection beams in the sample media are represented as $a_{i}$ and $a_{c}$, respectively. The angle at which the two 
beams intersect the main optical axis is denoted as $\theta$. A set of general dimensionless coordinates may be defined along the illumination and collection axes, as follows [19]:

$$
\begin{array}{ll}
\mathrm{u}_{\mathrm{i}}=\mathrm{k}_{\mathrm{i}} \mathrm{nz}_{\mathrm{i}} \sin ^{2} \alpha_{\mathrm{i}} & \mathrm{u}_{\mathrm{c}}=\mathrm{k}_{\mathrm{c}} \mathrm{nz}_{\mathrm{c}} \sin ^{2} \alpha_{\mathrm{c}} \\
\mathrm{v}_{\mathrm{i}}=\mathrm{k}_{\mathrm{i}} \mathrm{n} \sqrt{\mathrm{x}_{\mathrm{i}}^{2}+\mathrm{y}_{\mathrm{i}}^{2}} \sin \alpha_{\mathrm{i}} & \mathrm{v}_{\mathrm{c}}=\mathrm{k}_{\mathrm{c}} \mathrm{n} \sqrt{\mathrm{x}_{\mathrm{c}}^{2}+\mathrm{y}_{\mathrm{c}}^{2}} \sin \alpha_{\mathrm{c}}
\end{array}
$$

The wavenumbers for illumination and collection are defined as $k_{i}=2 \pi / \lambda_{i}$ and $k_{c}=2 \pi / \lambda_{c}$, respectively, where $\lambda_{\mathrm{i}}$ and $\lambda_{\mathrm{c}}$ are the wavelengths, and $\mathrm{n}$ is the index of refraction of the media.

The amplitude PSF describes the spatial distribution of the electric field of the focused beams. Diffraction theory may be used to show that the PSF of the illumination and collection beams is proportional to the Huygens-Fresnel integrals below [18]:

$$
\begin{aligned}
& \mathrm{U}_{\mathrm{i}}\left(\mathrm{v}_{\mathrm{i}}, \mathrm{u}_{\mathrm{i}}\right) \propto \int_{0}^{1} \mathrm{~W}_{\mathrm{i}}(\rho) \mathrm{J}_{0}\left(\rho \mathrm{v}_{\mathrm{i}}\right) \mathrm{e}^{-\mathrm{ju} \mathrm{u}_{\mathrm{i}} \rho^{2} / 2} \rho \mathrm{d} \rho \\
& \mathrm{U}_{\mathrm{c}}\left(\mathrm{v}_{\mathrm{c}}, \mathrm{u}_{\mathrm{c}}\right) \propto \int_{0}^{1} \mathrm{~W}_{\mathrm{c}}(\rho) \mathrm{J}_{0}\left(\rho \mathrm{v}_{\mathrm{c}}\right) \mathrm{e}^{-\mathrm{ju} \mathrm{u}_{\mathrm{c}} \rho^{2} / 2} \rho \mathrm{d} \rho
\end{aligned}
$$

where $\mathrm{J}_{0}$ is the Bessel function of order zero, and $\rho$ is a normalized radial distance variable at the objective aperture. The weighting function, $W(\rho)$, describes the truncation (apodization) of the beams. For uniform illumination, $W(\rho)=1$. For Gaussian illumination, the objectives truncate the beams at the $1 / \mathrm{e}^{2}$ intensity, resulting in a weighting function of $\mathrm{W}(\rho)=\mathrm{e}^{-\rho^{2}}$. In practice, the beams are typically truncated so that $99 \%$ of the power is transmitted. For a Gaussian beam with a radius (1/ $\mathrm{e}^{2}$ intensity) given by $\mathrm{w}$, an aperture with diameter $\pi \mathrm{w}$ passes $\sim 99 \%$ of the power. In this case, the weighting function is given as follows:

$$
\mathrm{W}(\rho)=\mathrm{e}^{-(\pi \rho / 2)^{2}}
$$

For the single axis configuration, the illumination and collection PSF's at the focal plane $\left(\mathrm{u}_{\mathrm{i}}=\right.$ $\mathrm{u}_{\mathrm{c}}=0$ ) are identical functions of the radial distance $\rho$, and can both be given by $\mathrm{U}_{\mathrm{s}}$ using the substitution $v=\mathrm{knr} \sin \alpha$, as follows:

$$
\mathrm{U}_{\mathrm{S}}(v) \propto \int_{0}^{1} \mathrm{~W}_{\mathrm{i}}(\rho) \mathrm{J}_{0}(\rho v) \rho d \rho
$$

The resulting signal at the detector $\mathrm{V}$ from a point source reflector in the media is proportional to the power received, and is given by the square of the product of the overlapping PSF's as follows:

$$
\mathrm{V}=\mathrm{A}\left|\mathrm{U}_{\mathrm{i}} \mathrm{U}_{\mathrm{c}}\right|^{2}
$$

where $A$ is a constant.

Similarly, since the depth of focus for each individual beam, described within the exponential term in the integral product of Eqs. (3) and (4), is much larger than that of the 
transverse width, the exponential term may be neglected. As a result, the detector output $\mathrm{V}_{\mathrm{d}}$ for the dual axes configuration for uniform illumination $(W=1)$, is given as follows:

$$
\mathrm{V}_{\mathrm{d}} \propto\left(\frac{2 \mathrm{~J}_{1}\left(\mathrm{v}_{\mathrm{i}}\right)}{\mathrm{v}_{\mathrm{i}}}\right)^{2}\left(\frac{2 \mathrm{~J}_{1}\left(\mathrm{v}_{\mathrm{c}}\right)}{\mathrm{v}_{\mathrm{c}}}\right)^{2}
$$

This expression can be combined with Eqs. (2) and (3) to derive the result for transverse and axial resolution with uniform illumination as follows [16]:

$$
\Delta \mathrm{x}_{\mathrm{d}}=\frac{0.37 \lambda}{\mathrm{n} \alpha \cos \theta} ; \quad \Delta \mathrm{y}_{\mathrm{d}}=\frac{0.37 \lambda}{\mathrm{n} \alpha} ; \quad \Delta \mathrm{z}_{\mathrm{d}}=\frac{0.37 \lambda}{\mathrm{n} \alpha \sin \theta}
$$

Note that for the dual axes configuration, the axial resolution is proportional to 1/NA, where $\mathrm{NA}=\mathrm{n} \sin \alpha \approx \mathrm{n} \alpha$, rather than $1 / \mathrm{NA}^{2}$, as is the case for the single axis design [3]. For example, with uniform illumination and the following parameters: $\alpha=0.21$ radians, $\theta=30$ degrees, $\lambda=0.785 \mu \mathrm{m}$ and $\mathrm{n}=1.4$ for tissue, Eq. (10) reveals a result for the dual axes configuration of $\Delta \mathrm{x}_{\mathrm{d}}=1.1 \mu \mathrm{m}, \Delta \mathrm{y}_{\mathrm{d}}=1.0 \mu \mathrm{m}$, and $\Delta \mathrm{z}_{\mathrm{d}}=2 \mu \mathrm{m}$ for the transverse and axial resolutions, respectively. Thus, sub-cellular resolution can be achieved in both the transverse and axial dimensions with the dual axes configuration using low NA optics but not with the single axis architecture.

For an endoscope-compatible instrument, delivery of the illumination and collection light is performed with use of optical fibers and is more appropriately modeled by a Gaussian rather than a uniform beam. With this apodization, the detector response for the dual axes configuration from a point source reflector in the media, given by Eq. (9), may be solved numerically as a function of transverse $\left(\mathrm{x}_{\mathrm{d}}\right.$ and $\left.\mathrm{y}_{\mathrm{d}}\right)$ and axial $\left(\mathrm{z}_{\mathrm{d}}\right)$ dimensions. The integrals are calculated in Matlab, and use the weighting function with $99 \%$ transmission. In comparison, this model reveals a result of $\Delta \mathrm{x}_{\mathrm{d}}=2.4 \mu \mathrm{m}, \Delta \mathrm{y}_{\mathrm{d}}=2.1 \mu \mathrm{m}$, and $\Delta \mathrm{z}_{\mathrm{d}}=4.2 \mu \mathrm{m}$ for the transverse and axial resolutions, respectively. Thus, the use of optical fibers, modeled by a Gaussian beam, produces results that are slightly worse but still comparable to that of uniform illumination [19].

\section{G. Dynamic range}

Differences in the dynamic range between the single and dual axes confocal configurations can also be illustrated with this model [18]. The calculated axial response for the single axis design with Gaussian illumination is shown by the dashed line in Fig. 6a, where optical parameters are used that achieve the same axial resolution (FWHM) of $4.2 \mu \mathrm{m}$. The result reveals that the main lobe falls off in the axial (z-axis) direction as $1 / z^{2}$, and reaches a value of approximately $-25 \mathrm{~dB}$ at a distance of $10 \mu \mathrm{m}$ from the focal plane $(\mathrm{z}=0)$. In addition, a number of side lobes can be appreciated.

In comparison, the response for the dual axes configuration, shown by the solid line in Fig. $6 \mathrm{a}$, reveals that the main lobe rolls off in the axial (z-axis) direction as $\exp \left(-\mathrm{kz}^{2}\right)$, and reaches a value of $-60 \mathrm{~dB}$ at a distance of $10 \mu \mathrm{m}$ from the focal plane $(\mathrm{z}=0)$. Thus, off-axis illumination and collection of light in the dual axes architecture results in a significant improvement in dynamic range and in an exponential rejection of out-of-focus scattered light in comparison to that for single axis. This advantage allows for the dual axes configuration to collect images with deeper tissue penetration and with a vertical crosssection orientation. The transverse response with Gaussian illumination is shown in Fig. 6b. 

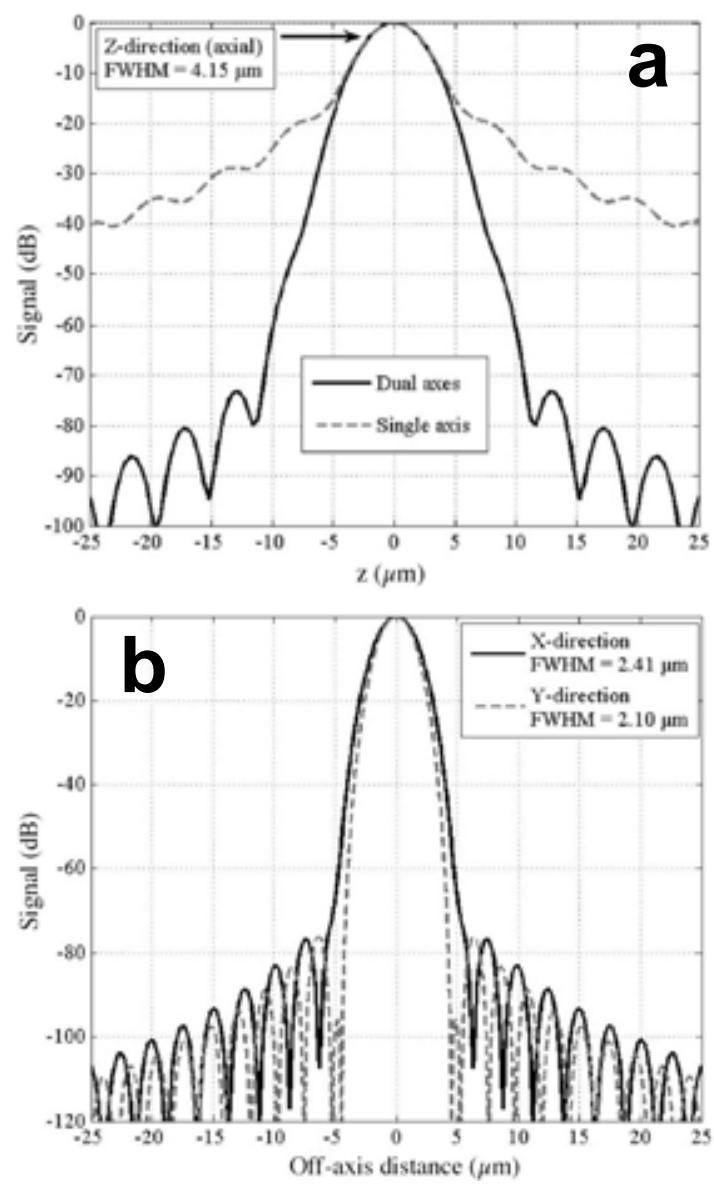

Fig. 6. Dynamic range of novel dual axes confocal architecture. a) The axial response of the single axis (dashed line) configuration falls off as $1 / z^{2}$ and that for the dual axes (solid line) design falls off as $\exp \left(-\mathrm{kz}^{2}\right)$, resulting in a significant improvement in dynamic range, allowing for vertical cross-sectional imaging to be performed. $b$ ) Transverse (X-Y direction) response.

\section{H. Post-objective scanning}

In confocal microscopes, scanning of the focal volume is necessary to create an image. In the single axis architecture, the high NA objectives used limit the working distance, thus the scan mirror is by convention placed on the pinhole (fiber) side of the objective, or in the preobjective position, as shown in Fig. 7a. Scanning orients the beam at various angles to the optical axis and introduces off-axis aberrations that expand the focal volume. In addition, the FOV of pre-objective scanning systems is proportional to the scan angle and the focal length of the objective. The diameter of the objective limits the maximum scan angle, and as this dimension is reduced for endoscope compatibility, the focal length and FOV are also diminished. 


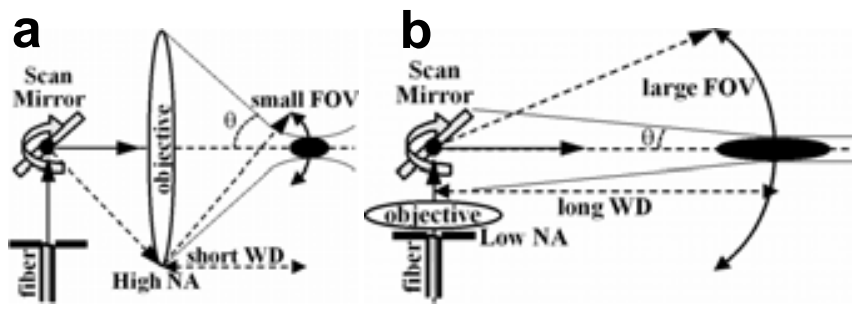

Fig. 7. a) For pre-objective scanning, illumination light is incident on the objective off-axis, resulting in more sensitivity to aberrations and limited FOV. b) With post-objective scanning, the incident light is on-axis, less sensitivity to aberrations, and large FOV. Postobjective scanning is made possible by the long WD produced by the low NA objectives used in the dual axes architecture.

In the dual axes configuration, the low NA objectives used creates a long working distance that allows for the scanner to be placed on the tissue side of the objective, or in the postobjective position [17]. This design feature is critical for scaling the size of the instrument down to millimeter dimensions for in vivo imaging applications without losing performance. As shown in Fig. 7b, the illumination light is always incident on-axis to the objective. In the post-objective location, the scan mirror can sweep a diffraction-limited focal volume over an arbitrarily large FOV, limited only by the maximum deflection angle of the mirror. Moreover, the scanner steers the illumination and collection beams together with the intersection of the two beams oriented at a constant angle $\theta$ and with the overlapping focal volume moving without changing shape along an arc-line. This property can be conceptualized by regarding the dual axes geometry as being equivalent to two separate beams produced from two circles in the outer annulus of a high NA lens containing a central obstruction (or a large central hole). A flat scan mirror deflects both beams equally, and thereby preserves the overlapping region without introducing aberrations to the beams.

\section{Improved rejection of scattering}

In the dual axes confocal architecture, the off-axis collection of light significantly reduces the deleterious effects of tissue scattering on the dynamic range of detection and allows for deeper ballistic photons to be resolved [20]. These features provide the unique capacity to collect vertical cross-sectional images in the plane perpendicular to the tissue surface. This is the preferred view of pathologists because differences from the normal patterns of tissue differentiation are revealed in the direction from the lumen to the sub-mucosa.

\section{Optical configurations}

The improvement in rejection of light scattered by tissue can be illustrated by comparing the dynamic range of detection between the single and dual axes optical configurations with equivalent axial resolution, as shown in Fig. $8 \mathrm{a}$ and $8 \mathrm{~b}$. The incident beams are modeled with a Gaussian profile because this is representative of light delivered through an optical fiber. For the single axis configuration, this beam is focused into the tissue by an ideal lens (L1). A mirror (M) is embedded in the tissue at the focal plane (parallel to the $x-y$ plane) of the objective lens. In this scheme, the rays that reflect from the mirror pass back through the lens L1, deflect at an angle off the beam splitter, and are focused by an ideal lens (L2) on to a pinhole detector. For the dual axes set-up, the incident Gaussian beam is focused into the 
tissue by an ideal lens (L3) with its axis oriented at an angle $\theta=30^{\circ}$ to the z-axis, and an ideal lens (L4) focuses the backscattered beam, with its axis $z^{\prime}$ at an angle $-30^{\circ}$ to the $z$-axis, onto the pinhole detector. As before, a mirror $(\mathrm{M})$ with its plane perpendicular to the $\mathrm{z}$-axis and passing through the coincident focuses of the lenses is embedded in the tissue to reflect the incident light to the detector. In both configurations, the lens system has a magnification of 1 from the focal plane to the pinhole detector.
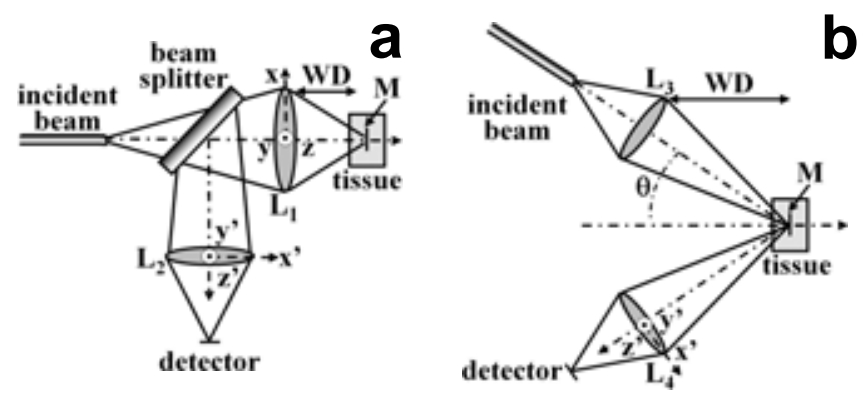

Fig. 8. a) single axis and b) dual axes optical configurations are used to evaluate the axial response at the detector.

In order to achieve an equivalent $-3 \mathrm{~dB}$ axial resolution (FWHM), the NA's for the single and dual axes configurations are defined to be 0.58 and 0.21 , respectively. From diffraction theory, discussed above, the theoretical transverse and axial resolutions for the PSF for dual axes at a wavelength $\lambda=633 \mathrm{~nm}$ with an average tissue refractive index of 1.4 and NA $=0.21$ are found to be $\Delta \mathrm{x}=1.16 \mu \mathrm{m}, \Delta \mathrm{y}=1.00 \mu \mathrm{m}$, and $\Delta \mathrm{z}=2.00 \mu \mathrm{m} .3$ The mirror is placed at a distance of $200 \mu \mathrm{m}$ below the tissue surface in the focal plane of the objective lenses for both the single and dual axes configurations. This depth is representative of the imaging distance of interest in the epithelium of hollow organs. The calculations performed to analyze the effects of tissue scattering on light are based on Monte Carlo simulations using a nonsequential ray tracing program (ASAP® 2006 Breault Research Organization, Tucson, AZ). Three assumptions are made in this simulation study: 1) multiple scattering of an incoherent beam dominates over diffraction effects, 2) the non-scattering optical medium surrounding the lenses and the tissue (the scattering medium) is index matched to eliminate aberrations, and 3) absorption is not included to simplify this model and because there is much larger attenuation due to the scattering of ballistic photons.

\section{Mie scattering analysis}

We use Mie theory with the Henyey-Greenstein phase function $p(\theta)$ to model the angular dependence of tissue scattering, as follows [21, 22]:

$$
p(\theta)=\frac{1}{4 \pi} \frac{1-g^{2}}{\left(1+g^{2}-2 g \cos \theta\right)^{3 / 2}}
$$

where $g$, the anisotropy factor, is defined as

$$
g=\langle\cos \theta\rangle=\int_{0}^{2 \pi} \int_{0}^{\pi} \cos \theta \cdot p(\theta) \sin \theta d \theta d \varphi .
$$



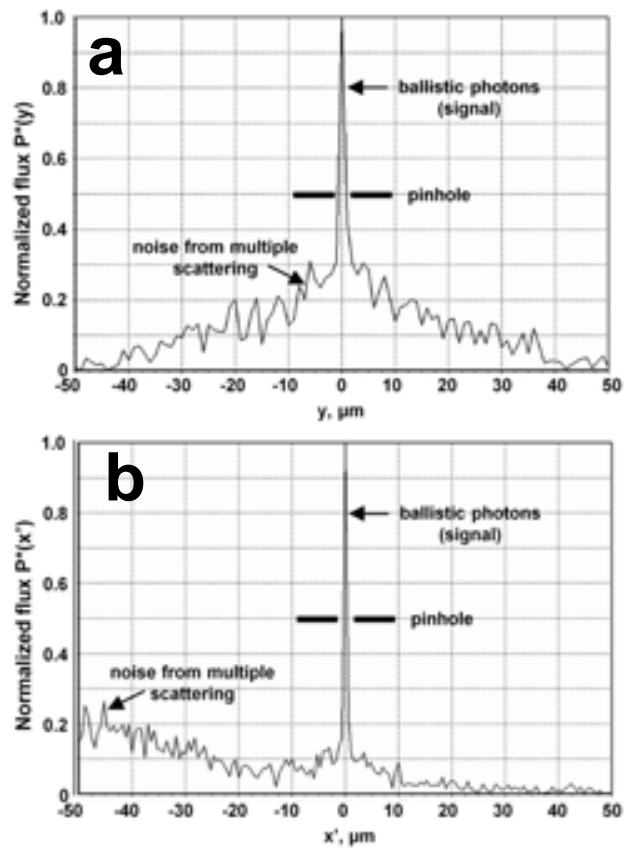

Fig. 9. Distributions of photon flux in tissue scattering model. The peak value of multiple scattered photons for A) single axis is co-located with the confocal pinhole while that for B) dual axes is separated by $\sim 50 \mu \mathrm{m}$. As a consequence, the ballistic photons for dual axes result in a greater signal-to-noise ratio.

Given the average scatterer size, refractive index, and concentration, the attenuation coefficient $\mu_{\mathrm{s}}$ and anisotropy $g$ are determined and provided to the ASAP program as simulation parameters. For a tissue phantom composed of polystyrene spheres with a diameter of $0.48 \mu \mathrm{m}$, refractive index 1.59 , and a concentration of 0.0394 spheres $/ \mu \mathrm{m}^{3}$ in water, the values $\mathrm{g}=0.81$ and $\mu_{\mathrm{s}}=5.0 \mathrm{~mm}^{-1}$ at $\lambda=633 \mathrm{~nm}$ are calculated from Mie theory [23]. For single axis, $\mathrm{P}\left(\mathrm{y}^{\prime}\right)$ is defined as the photon flux distribution along the $\mathrm{y}^{\prime}$-axis at the detector. The photon flux can be normalized by defining $\mathrm{P}^{*}\left(\mathrm{y}^{\prime}\right)=\mathrm{P}\left(\mathrm{y}^{\prime}\right) / \mathrm{P}_{\max }$, where $\mathrm{P}_{\max }$ is the maximum flux. The normalized flux $\mathrm{P}^{*}\left(\mathrm{y}^{\prime}\right)$ consists of ballistic (signal) and multiple scattered (noise) photons, as shown in Fig. 9a [24]. The maximum flux for both the signal and noise components arrive at center of the detector. A confocal pinhole placed in front of the detector can filter out some but not all of this "noise," resulting in a reduced signal-tonoise ratio (SNR). For dual axes, the detector is angled off the optical axis by 30 deg. $P\left(x^{\prime}\right)$ is defined as the photon flux distribution along the $x^{\prime}$-axis at the detector. The photon flux can be normalized by defining $\mathrm{P}^{*}\left(\mathrm{x}^{\prime}\right)=\mathrm{P}\left(\mathrm{x}^{\prime}\right) / \mathrm{P}_{\max }$, where $\mathrm{P}_{\max }$ is the maximum flux at the detector. Fig. $9 \mathrm{~b}$ shows that normalized photon flux distribution for dual axes also exhibits a ballistic and multiple scattered components. However, for dual axes, the peak flux of multiple scattered photons arrives $\sim 50 \mu \mathrm{m}$ lateral to the center of the detector where the ballistic photons arrive, a consequence of off-axis collection. Thus, there is much less "noise" for the confocal pinhole (diameter $\sim 1 \mu \mathrm{m}$ ) to filter out, resulting in a higher SNR and dynamic range. 


\section{Improvement in dynamic range}

An implication of this result is that the dual axes configuration has improved dynamic range compared to that of single axis. This difference can be quantified by determining the axial response at the detector. This can be done by calculating the photon flux $f(\Delta z)$ as the mirror is displaced along the $z$-axis in the tissue. The flux is calculated using Monte-Carlo simulations in ASAP with the mirror at positioned in the range $-10 \mu \mathrm{m}<\Delta z<10 \mu \mathrm{m}$ with respect to the focal plane at $z=0$, which is located at $200 \mu \mathrm{m}$ below the tissue surface. The flux is then normalized according to $F(\Delta z)=f(\Delta z) / f(0)$. The axial response is shown in Fig. 10a for various pinhole diameters $\mathrm{D}$, including 1,2 and $3 \mu \mathrm{m}$, which correspond to typical fiber core dimensions. Note that for each pinhole diameter, the dual axes (DA) configuration has significantly better dynamic range than that of single axis (SA). Note that the introduction of tissue scattering results in a reduction of the dynamic range compared to that found in free space, as shown by Fig. 6 a.
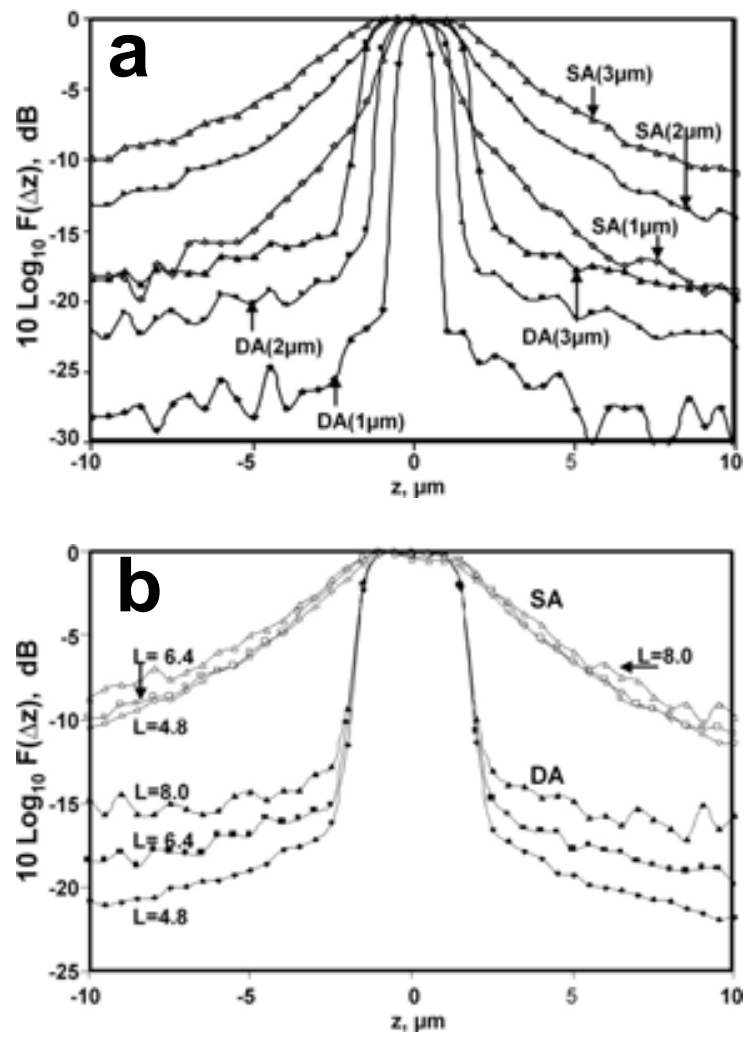

Fig. 10. Axial response for single and dual axes geometries. The dual axes (DA) configuration has a much greater dynamic range than that for single axis (SA) given different a) pinhole diameters $(1,2$, and $3 \mu \mathrm{m})$ and $b)$ optical lengths $L(4.8,6.4$ and 8.0).

We can also determine the axial response of the detector for various optical lengths in tissue. This analysis reveals differences in the dynamic range between the single and dual axes 
configuration for tissues with various scattering properties. The total optical length $\mathrm{L}$ is defined as twice the product of the scattering coefficient $\mu_{\mathrm{s}}$ and the tissue depth $\mathrm{t}$, or $\mathrm{L}=$ $2 \mu_{\mathrm{s}} \mathrm{t}$. The factor of two originates from the fact that the total path length is twice the tissue depth. The axial response is shown in Fig. 10b for various optical lengths L, including 4.8, 6.4, and 8.0. Note that for each optical length $\mathrm{L}$, the dual axes (DA) configuration has significantly better dynamic range than that of single axis (SA). These values of $L$ are typical parameters of gastrointestinal epithelium. At $\lambda=633 \mathrm{~nm}, \mu_{\mathrm{s}}$ is about $7 \mathrm{~mm}^{-1}$ for esophagus tissue [24] and about $20 \mathrm{~mm}^{-1}$ for normal colon mucosa [25]. The range of tissue depths spanned by $\mathrm{L}=4.8$ to 8 for esophagus and colon is $340 \mu \mathrm{m}$ to $570 \mu \mathrm{m}$ and $120 \mu \mathrm{m}$ to $200 \mu \mathrm{m}$, respectively. In addition, these results shows that for single axis only minimal changes occur in the dynamic range with approximately a factor of 2 difference in optical thickness L, while for dual axes significant changes occur over this thickness range. Furthermore, scattering does not appear to alter the FWHM of the axial response for either single or dual axes over this range of lengths.

\section{Geometric differences produced by off-axis detection}

The superior axial response of the dual axes confocal architecture has a simple geometric explanation. When the mirror moves away from the focal plane by $\pm \Delta$, the centroid of the beam is steered away from the optical axis by $\pm 2 \Delta \sin \theta$ from where the center of the pinhole is located [20]. Even taking into consideration diffraction and the broadening of the out-offocus beam, the beam intensity decreases exponentially when $\Delta>\mathrm{D} / 2$ (for $\theta=30^{\circ}$ ). But in the single axis case, many of the photons scattered near the vicinity of the focal plane $( \pm \Delta)$ are collected by the detector through the pinhole. Thus, the spatial filtering effect by a pinhole for the single axis configuration is not as effective as that for dual axes. The implication of this effect for imaging deep in tissue is evident. In the single axis case, many of the multiple scattered photons that arrive from the same direction as that of the ballistic photons, starting from the surface to deep within the tissue, are collected by the detector despite the presence of a pinhole to filter the out-of-focus light. This explains why in Fig. 9a the single axis configuration has a large noise component alongside the ballistic component. Thus, the dual axes confocal architecture provides optical sectioning capability that is superior to that of the conventional single axis design in terms of SNR and dynamic range, and this result can be generalized to a range of relevant pinhole sizes. As a result, the dual axes architecture allows for imaging with greater tissue penetration depth, thus is capable of providing images in the vertical cross-section with high contrast. The implementation of the dual axes confocal configuration to an endoscope compatible instrument for collection of both reflectance and fluorescence has significant implications for in vivo imaging by providing both functional and structural information deep below the tissue surface.

\section{Tabletop dual axes confocal imaging instruments}

The dual axes confocal architecture was first implemented as a tabletop instrument using readily available optical components to demonstrate the proof of concept of off-axis illumination and collection with post-objective scanning. In particular, the primary advantages of the dual axes configuration including high dynamic range and deep tissue penetration are revealed by vertical cross-sectional images with either reflectance or fluorescence. The combination of these two imaging modes forms a powerful strategy for integrating structural with functional information. 
The dual axes optical design incorporates a solid immersion lens (SIL) made from a fusedsilica hemisphere at the interface where the two off-axis beams meet the tissue. This refractive element minimizes spherical aberrations that occur when light undergoes a step change in refractive index between two media. The curved surface of the SIL provides a normal interface for the two beams to cross the air-glass boundary. Fused silica is used because its index of refraction of $n=1.45$ is closely matched to that of tissue. Note that as the beams are scanned away from their neutral positions, they will no longer be incident to the surface of the SIL and small aberrations will occur. Another feature of the SIL is that its curved surface increases the effective NA of the beams in the tissue by a factor of $n$, the index of refraction, and produces higher resolution and light collection efficiency. On the other hand, the SIL acts to reduce the scanning displacement of the beams in the tissue by a factor of $1 / n$ so that larger deflections are needed to achieve the desired scan range.

J. Horizontal cross-sectional imaging instrument
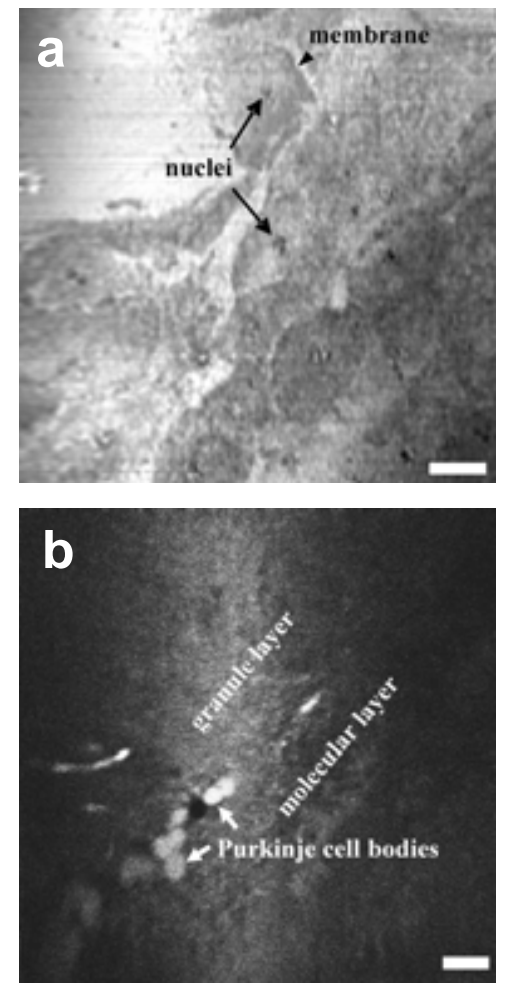

Fig. 11. Horizontal cross-sectional dual axes images ex vivo. a) Squamous esophageal mucosa collected at $\mathrm{z}=0 \mu \mathrm{m}$ with $\lambda=488 \mathrm{~nm}$ reveals sub-cellular features, including cell nuclei (arrows) and membrane (arrowhead), scale bar $20 \mu \mathrm{m}$. b) Normal colonic mucosa at z $=150 \mu \mathrm{m}$ with $\lambda=1.3 \mu \mathrm{m}$ illumination reveals circular crypts with colonocytes surround the lumen and lamina propria (LP) filling space in between the crypts, scale bar $50 \mu \mathrm{m}$.

Reflectance imaging takes advantage of subtle differences in the refractive indices of tissue micro-structures to generate contrast. The backscattered photons can provide plenty of 
signal to overcome the low NA objectives used for light collection in the dual axes configuration. The first reflectance images were collected with a tabletop system that used a $488 \mathrm{~nm}$ semiconductor laser that delivered illumination into a single mode optical fiber that was focused by a set of collimating lenses with $\mathrm{NA}=0.16$ to a spot size with $\sim 400 \mu \mathrm{W}$ of power [16]. These parameters produced a transverse and axial resolution of 1.1 and $2.1 \mu \mathrm{m}$, respectively. The reflected light was collected by a complementary set of optics. The off-axis illumination and collection was performed at $\theta=30^{\circ}$ to the main optical axis. Reflectance images were collected in horizontal cross-sections of freshly excised specimens of esophagus ex vivo. As shown in Fig. 11a, the cell membrane and individual nuclei of squamous (normal) esophageal mucosa can be appreciated in the image collected at $\mathrm{z}=0 \mu \mathrm{m}$, scale bar $20 \mu \mathrm{m}$.

Much greater image contrast can be achieved with fluorescence imaging where the use of optical reporters, such as GFP, and exogenous probes can reveal over expression of molecular targets. The same tabletop dual axes microscope was also used to collect fluorescence images with a long pass filter to block the excitation light and photomultiplier tube (PMT) for detection [26]. In Fig. 11b, a fluorescence image of the cerebellum of a transgenic mouse that constituitively expresses GFP under the control of a $\beta$-actin promoter at a depth of $\mathrm{z}=30 \mu \mathrm{m}$ is shown, scale bar $50 \mu \mathrm{m}$. Purkinje cell bodies (arrows) can be seen as large round structures aligned side by side in a row, separating the granule layer and the molecular layer.

\section{K. Vertical cross-sectional imaging instrument}

\section{Reflection imaging mode}

In order to collect vertical cross-sectional images, heterodyning can be used to provide a coherence gate that filters out illumination photons that are multiply-scattered and travel over longer optical paths within the tissue [17]. This approach is demonstrated with a fiber optic Mach-Zehnder interferometer, shown in Fig. 12a. A broadband near-infrared source produces light centered at $\lambda=1345 \mathrm{~nm}$ with a $3 \mathrm{~dB}$ bandwidth of $35 \mathrm{~nm}$ and a coherence length in tissue of $\sim 50 \mu \mathrm{m}$. A fiber coupler directs $\sim 99 \%$ of the power to the illumination path, which consists of a single mode optical fiber $\left(\mathrm{SMF}_{1}\right)$ with a collimating $\left(\mathrm{CL}_{1}\right)$ and focusing lens $\left(\mathrm{FL}_{1}\right)$ with $\mathrm{NA}=0.186$. The axes of illumination and collection are oriented at $\theta=30^{\circ}$ to the midline. Light reflected from the tissue is collected by the second set of focusing $\left(\mathrm{FL}_{2}\right)$ and collimating $\left(\mathrm{CL}_{2}\right)$ lenses into another single mode fiber $\left(\mathrm{SMF}_{2}\right)$. The lens and fiber parameters are the same for both the illumination and collection beams. The fiber optic coupler directs $\sim 1 \%$ of the source into a reference beam which is frequency shifted by an acousto-optic modulator at $55 \mathrm{MHz}$ for heterodyne detection. An adjustable optical delay is used to increase the signal by matching the optical path length of the reference beam to that of the ballistic photons. An adjustable optical delay is used to increase the signal. In addition, a polarization controller consisting of two half-wave plates and a single quarterwave plate is used to maximize the signal. The reference and collection beams are combined by a $50 / 50$ coupler and the resulting heterodyne signal is detected by a balanced InGaAs detector $\left(D_{1}, D_{2}\right)$ with a bandwidth of $80 \mathrm{MHz}$. The resulting electronic signal is then processed with a band pass filter (BPF) with a $3 \mathrm{MHz}$ bandwidth centered at $55 \mathrm{MHz}$, then demodulated (DM), digitized by a frame grabber (FG), and displayed (D).

In this heterodyne detection scheme, the reference beam essentially provides amplification of the weak collection beam via coherent optical mixing, and enables the measurement of reflected light with a dynamic range larger than $70 \mathrm{~dB}$. Post-objective scanning is performed with the scan mirror (SM) placed distal to the objective lenses. Reflectance images were 
collected from fresh biopsy specimens taken from the squamo-columnar junction of subjects with Barrett's esophagus. Specimens with dimensions of $\sim 3 \mathrm{~mm}$ were resected with jumbo biopsy forceps, and the mucosal surface was oriented normal to the z-axis. Vertical crosssectional images were collected with depth of $1 \mathrm{~mm}$. From Fig. 12b, squamous (normal) mucosa is present over the left half of the image with an intact epithelium (EP). The other structures of normal esophageal mucosa, including the muscularis mucosa (MM), submucosa (SM), and muscularis propria (MP), can also be identified. Columnar mucosa consistent with intestinal metaplasia is seen over the right half of the image, and reveals the presence of pit epithelium (PE) [17]. These findings correlate with the tissue microstructures seen on histology.

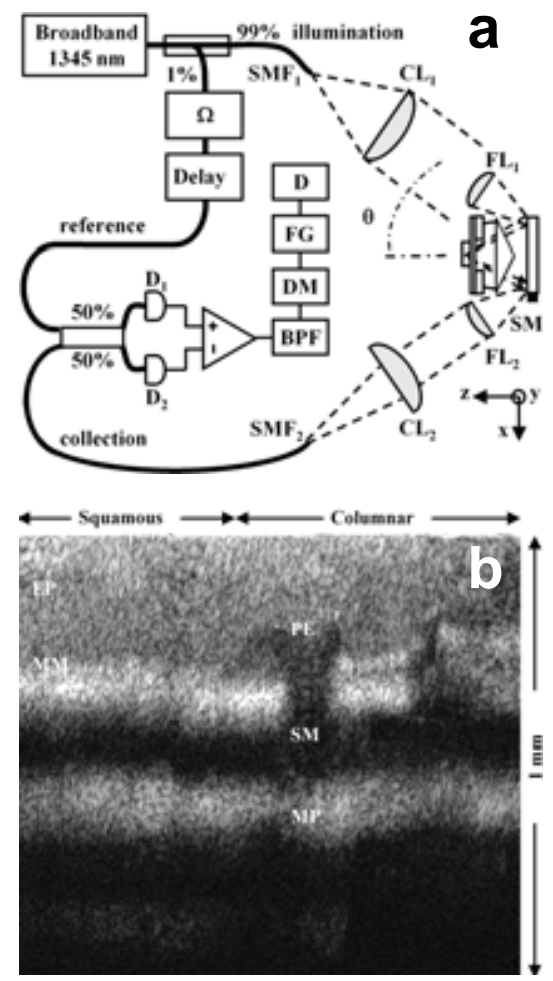

Fig. 12. Vertical cross-sectional dual axes confocal reflectance images ex vivo. a) Schematic of optical circuit for heterodyne detection, details discussed in the text. b) Reflectance image of squamo-columnar junction in esophagus with vertical depth of $1 \mathrm{~mm}$. Squamous (normal) mucosa reveals epithelium (EP) and muscularis mucosa (MM) over left half. Columnar (intestinal metaplasia) mucosa shows pit epithelium (PE) over right half. Submucosa (SM) and musclaris propria (MP) are seen on both sides.

\section{Fluorescence imaging mode}

Fluorescence detection adds an entirely new dimension to the imaging capabilities of the dual axes architecture. Detection in this mode offers an opportunity to achieve much higher image contrast compared to that of reflectance and is sensitive to labeled molecular probes 
that can identify specific tissue and cellular targets. These features provide a method to perform functional as well as structural imaging, and allows for the study of a wide variety of molecular mechanisms. Although the use of low NA objectives in the dual axes configuration reduces the collection efficiency of the optics by a factor of $\sim \mathrm{NA}^{2}$, this deficit can be overcome by the use of bright fluorophores. In order to achieve the deep tissue penetration depths possible with the off-axis collection of light, a near-infrared laser at 785 $\mathrm{nm}$ is used as the source and a PMT with a long pass filter to block the excitation light is used as the detector [27]. The large dynamic range $(>40 \mathrm{~dB})$ of the dual axes confocal architecture encountered with collection of vertical cross-sectional images requires modulation of the PMT gain to compensate for the rapid decrease in fluorescence signal with axial depth due to tissue absorption and scattering.

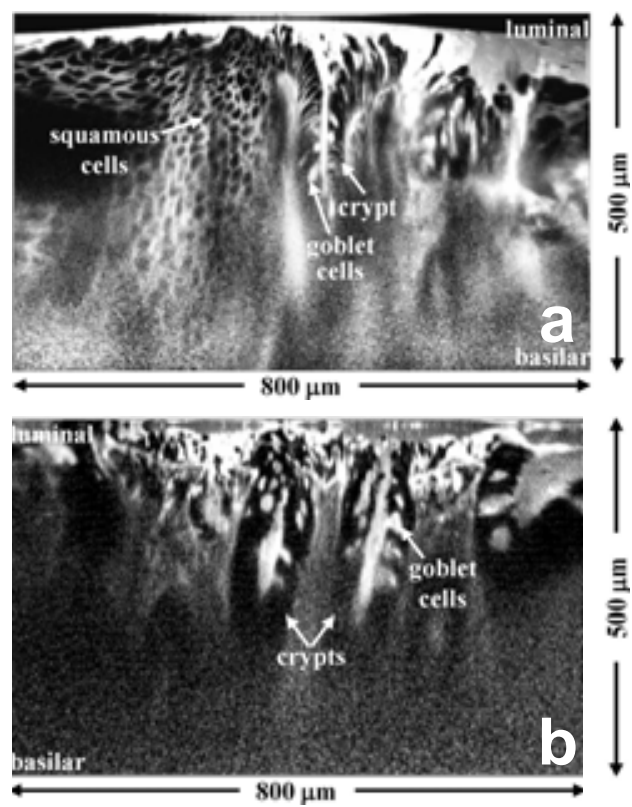

Fig. 13. Vertical cross-sectional dual axes confocal fluorescence images ex vivo. a) Squamocolumnar junction in esophagus with vertical depth of $500 \mu \mathrm{m}$. Squamous mucosa present over left half. Columnar (intestinal metaplasia) mucosa over right half shows crypts with goblet cells. b) Colon. Many goblet cells can be seen in dysplastic crypts from a flat colonic adenoma.

In Fig. 13, vertical cross-sectional fluorescence images of a) esophagus and b) colon collected with a tabletop dual axes confocal microscope are shown [27]. These specimens were incubated with a near-infrared dye, LI-COR IRDye ${ }^{\circledR} 800$ CW NHS Ester (LI-COR Biosciences, Inc) prior to imaging after being freshly excised during endoscopy. These images were collected at 2 frames per second with a transverse and axial and resolution of 2 and $3 \mu \mathrm{m}$, respectively. With use of post-objective scanning, a very large FOV of $800 \times 500$ $\mu \mathrm{m}^{2}$ deep was achieved. In Fig. 13a, the specimen was collected from the squamo-columnar junction of a patient with Barrett's esophagus. Over the left half of the image, the individual squamous cells from normal esophageal mucosa can be seen in the luminal to the basilar 
direction over a depth of $500 \mu \mathrm{m}$. Over the right half of the image, vertically oriented crypts with individual mucin-secreting goblet cells associated with intestinal metaplasia can be appreciated as brightly stained vacuoles. This diseased condition is associated with greater than 100 fold relative risk of developing cancer in the esophagus. In Fig. 13b, the specimen was collected from a flat colonic adenoma, and the image reveals vertically oriented dysplastic crypts with individual goblet cells.

Volume rendering can also be performed with the dual axes confocal microscope to illustrate three-dimensional (3D) imaging capabilities. These views are important for tracking cell movements, observing protein-protein interactions, and monitoring angiogenic development. A xenograft mouse model of glioblastoma multiforme has been developed by subcutaneously implanting $\sim 10^{7}$ human U87MG glioblastoma cells in the flank of a nude mouse. Horizontal cross-sectional fluorescence images were collected with a tabletop instrument when the tumors reached $\sim 1 \mathrm{~cm}$ in size. The mice were anesthetized for the in vivo imaging session, and indocyanine green (ICG) at a concentration of $0.5 \mathrm{mg} / \mathrm{ml}$ was injected intravenously to produce contrast. A skin flap overlying the tumor was exposed, and horizontal cross-sectional images were collected with a FOV of $400 \times 500 \mu \mathrm{m}^{2}$. A fluorescence image collected at $50 \mu \mathrm{m}$ below the tissue surface, shown in Fig. 14a, reveals that the glioblastoma has developed a dense, complex network of tortuous vasculature. A total of 400 horizontal cross-sectional images acquired at $1 \mu \mathrm{m}$ increments were used to
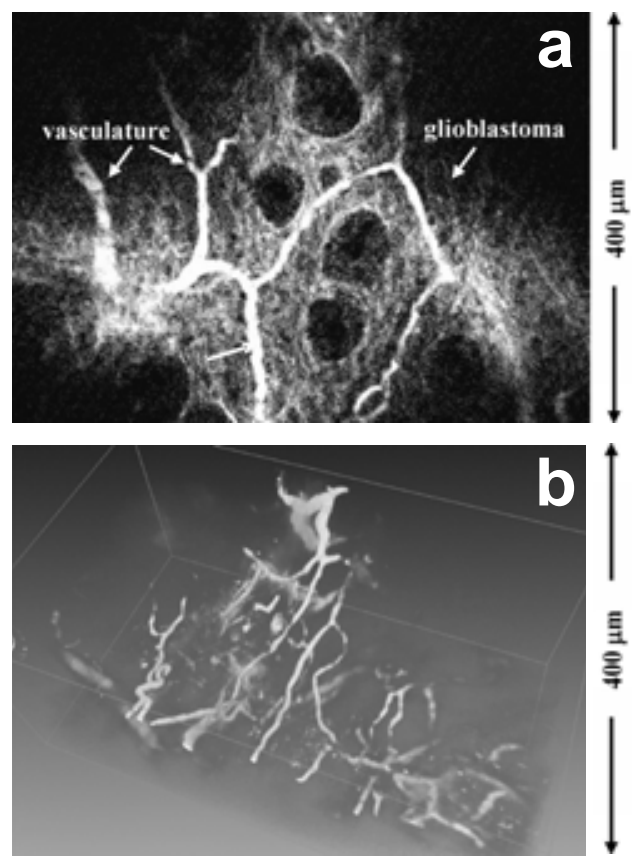

Fig. 14. Dual axes confocal fluorescence images in small animal models. a) A horizontal cross-sectional fluorescence image of a human U87MG xenograft glioblastoma tumor implanted subcutaneously in the flank of a nude mouse was collected in vivo at $50 \mu \mathrm{m}$ depth using i.v. indocyanine green (ICG). b) A 3D volumetric image is generated from a zstack of 400 sections collected at $1 \mu \mathrm{m}$ intervals. 
generate the 3D volumetric image, shown in Fig. 14b. Volume rendering was performed using AmiraTM modeling software.

\section{Mems-scanner based dual axes confocal imaging instruments}

The long working distance created by the low NA objectives in the dual axes architecture provides space for a scanning mechanism to be placed in the post-objective position. This location is a key feature of the design that allows for scaling down of the optics to millimeter dimensions. Moreover, for in vivo imaging, a fast scan rate is needed to overcome motion artifacts introduced by organ peristalsis, heart beating, and respiratory activity, typically requiring a frame rate of $>4$ per second. As a result, we have developed a MEMS-scanner based miniature and endoscope compatible imaging instruments without loss of performance. This strategy is much more complex than other approaches being developed, but is well suited to meet the size and speed requirements for in vivo imaging in a compact package $[28,29]$.

\section{MEMS Scanner Structure}

The schematic of the 2-D MEMS scanner is shown in Fig. 15a. It has a gimbal structure, and is electrostatically actuated by self-aligned, vertical combdrives to give large deflection. The mirror can be actuated with respect to the frame by rotating around the springs that define the inner axis. The frame supporting the mirror can be actuated with respect to the substrate by rotating around the springs that define the outer axis. Fig. $2 \mathrm{~b}$ shows the cross-sections of various structures of the device, which is made in double-stacked silicon-on-insulator (SOI). The two device layers are each $30 \mu \mathrm{m}$ thick with an oxide layer of $0.38 \mu \mathrm{m}$ in between. The substrate thickness is $530 \mu \mathrm{m}$, while the oxide layer between the lower device layer and substrate has a thickness of $1 \mu \mathrm{m}$. The thick device layers increase the tilt range of the mirror by deeper comb engagement, and lead to a larger FOV. The mirror, movable combteeth, and inner torsional springs are fabricated in the upper device layer. The fixed combteeth, outer torsional spring, and frame consist of double-stacked layers. A backside window is located below the gimbal structure to release the device and allow large-range motion. Four actuation voltages are supplied to the lower layer to actuate both sides of each axis (outer: V1 and V2, inner: V3 and V4, in Fig. 15a). The upper layer and substrate are both grounded. Electrical isolation between the device layers and the substrate is provided by buried oxide layers, as seen in Fig. 15b. The double-stacked layers of the outer torsional spring and frame deliver actuation voltages and ground to the inner combdrives.

Alignment of the off-axis illumination and collection are achieved with two mirrors connected together by a strut. The size of the mirrors $(600 \mu \mathrm{m} \times 650 \mu \mathrm{m})$ and the distance between them $(1.51 \mathrm{~mm})$ enable an off-axis half-angle, $\theta$, of $24.3^{\circ}$. The inner combdrives are placed on the connecting beam between the two mirrors and the inner springs are recessed into the mirror sides, to allow the die size to be reduced to $3.4 \mathrm{~mm} \times 2.9 \mathrm{~mm}$ to fit inside the scanhead package. The frame width is designed to be $150 \mu \mathrm{m}$ to prevent stress-induced curvature of the gimbal. In order to increase the torque with the same number of combs, the moment arm is lengthened by placing the outer combdrives further away from the outer torsional spring.

\section{Theoretical Analysis}

The outer spring consists of two silicon device layers (each $30 \mu \mathrm{m}$ thick) with an oxide layer $(0.38 \mu \mathrm{m})$ in between, and delivers three different voltages to the inner frame. Its dimensions 


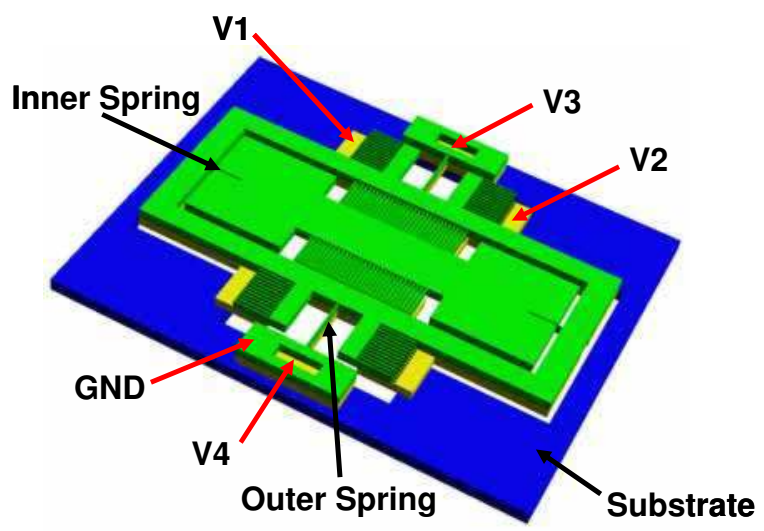

a

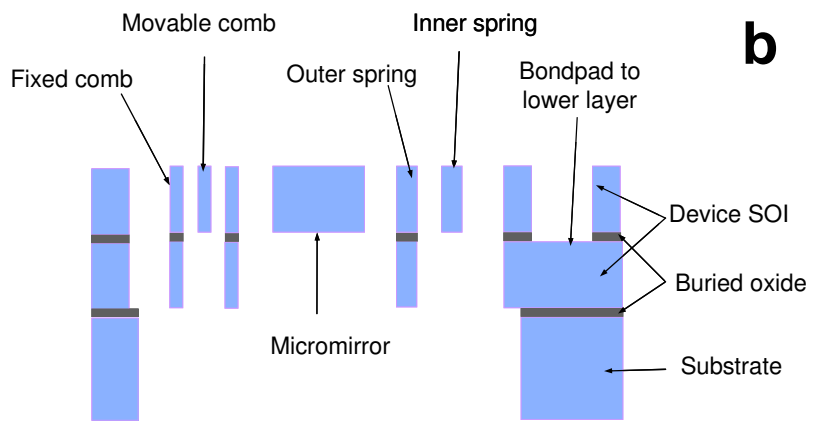

Fig. 15. a) Schematic drawing of the 2-D MEMS scanner. b) Cross-sectional view of various structures of the scanner.

are $60.38 \mu \mathrm{m}$ thick and $350 \mu \mathrm{m}$ long. The inner spring consists of one silicon layer and is therefore $30 \mu \mathrm{m}$ thick. The mechanical torque of the torsional spring can be expressed as

$$
T_{\phi}(\phi)=k_{\phi}(\phi),
$$

where $k_{\phi}$ is the torsional spring constant, and $\phi$ is the mechanical deflection angle. Both torsional springs are rectangular, so $k_{\phi}$ is given as follows [30].

$$
k_{\phi}=\frac{2 G}{3} \frac{t w^{3}}{l}\left[1-\frac{192}{\pi^{5}} \frac{w}{t} \tanh \left(\frac{\pi}{2} \frac{t}{w}\right)\right], \text { for } w<t
$$

Here $G$ is the shear modulus given by $G=E / 2(1+v)$, where $E$ is the Young's modulus, and $v$ is Poisson's ratio. The parameters $l, w, t$ represent the length, width, and thickness of the spring, respectively.

When an electrical voltage, $V$, is applied between the fixed and movable combs, the electrical torque, $T_{e}$, is given as follows. 


$$
T_{e}(\phi, V)=N V^{2} \frac{\partial C_{u n i t}(\phi)}{\partial \phi}
$$

Here $N$ is the number of comb pairs, and $C_{u n i t}$ is the capacitance of a comb pair. In steadystate, the mechanical torque of the spring is balanced by the electrostatic torque of the combdrives, and torques expressed by equations (13) and (15) are equal. A hybrid program which combines a 2-D finite element method (FEM) with analytical calculation [31] is used for generating the simulated DC transfer curves of Fig. 16.

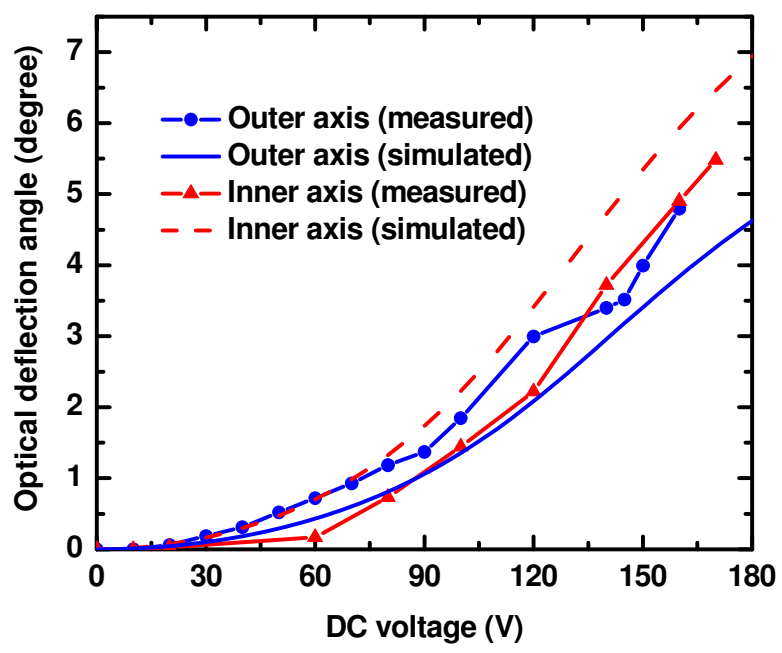

Fig. 16. Static optical deflection curve of MEMS scanner.

N. Device Fabrication

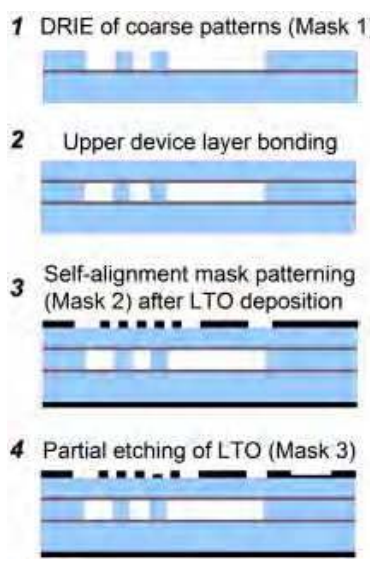

5

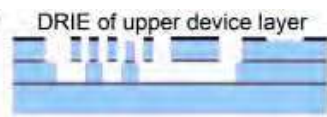

6 DRIE of lower device layer by Mask 2 and upper device layer by Mask 3

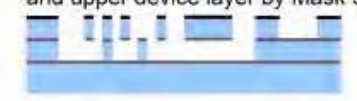

7 Backside patterning and DRIE for release (Mask 4)

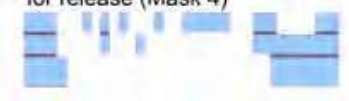

Single crystal silicon (SCS)

Thermal oxide

Low temperature oxide (LTO)

Fig. 17. MEMS scanner fabrication process flow.

The design of this mirror uses a gimbal geometry to perform scanning in the horizontal (X$\mathrm{Y})$ plane, and rotation around an inner and outer axes defined by the location of the 
respective springs. The overall structure has a barbell shape with two individual mirrors that have active surface dimensions of $600 \times 650 \mu \mathrm{m}^{2}$. A $1.51 \mathrm{~mm}$ long strut connects these two mirrors so that the illumination and collection beams preserve the overlapping focal volume in the tissue. The fabrication process flow, shown in Fig. 17, starts with a SOI wafer composed of a silicon substrate, buried oxide, and silicon lower device layers that are 530, 0.38, and $30 \mu \mathrm{m}$ thick, respectively [32]. A deep-reactive-ion-etch (DRIE) of coarse patterns, including the combdrives and trenches, is performed on the SOI wafer with Mask 1 (step 1). Next, an oxide layer is grown on a plain silicon wafer using a wet oxidation process. This wafer is then fusion bonded onto the etched surface of the SOI wafer (step 2). The yield is increased by bonding in vacuum, and the bonded plain wafer is ground and polished down to $30 \mu \mathrm{m}$ thickness, forming the upper device layer.

The two oxide layers between the silicon layers provide electrical isolation, and act as etch stops, allowing for precise thickness control. The front side of the double-stacked SOI wafer is patterned and DRIE etched to expose the underlying alignment marks in the lower device layer. Then, a low temperature oxide (LTO) layer is deposited on both sides of the wafer. The front side layer is patterned by two masks. The first mask (Mask 2) is the self-alignment mask (step 3), and is etched into the full thickness of the upper LTO layer. The second mask (Mask 3) is mainly for patterning the electrodes for voltage supplied to the lower device layer (step 4). It goes through a partial etch leaving a thin layer of LTO. The alignment accuracy of each step needs to be $>g / 2$, where $g$ is the comb gap. Since most devices have $6 \mu \mathrm{m}$ comb gaps, this leads to a required alignment accuracy of better than $3 \mu \mathrm{m}$.

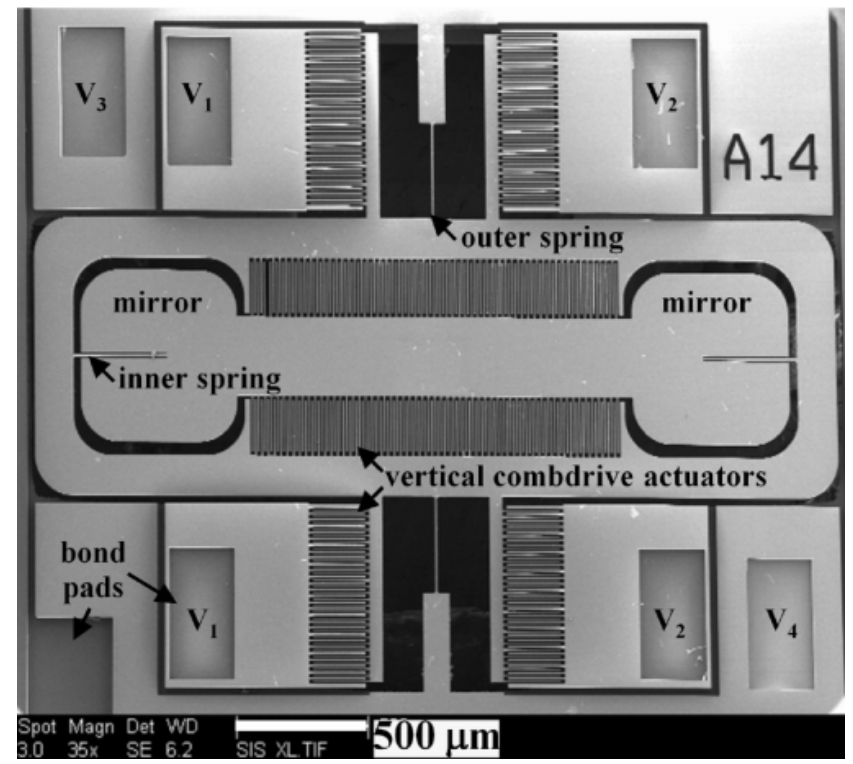

Fig. 18. SEM of 2D gimbaled MEMS scanner, scale bar $500 \mu \mathrm{m}$.

Good alignment accuracy is important to minimize failures due to electrostatic instability during actuation. These three masks eventually define the structures in the upper, lower, and double-stacked layers. After the front side patterning is done, the LTO layer on the wafer back side is stripped (step 5). The wafer is cleaned and photoresist is deposited on the 
back side. Then, front side alignment marks are patterned. Next, the upper silicon layer is etched with the features of Mask 2 in DRIE. Then, a thin LTO and buried oxide layer is anisotropically dry-etched. Finally, the lower and upper silicon layers are etched (DRIE) simultaneously with features patterned by Mask 2 and 3, respectively (step 6).

For backside processing, the wafer is bonded to an oxidized handle wafer with photoresist. The back side trenches are patterned with Mask 4 on photoresist (step 7). The back side trench should etch through the substrate to release the gimbal structure, so handle wafer bonding and thick resist is required for DRIE. Alignment to the front side features are accomplished by aligning to the previously etched patterns. After the substrate $(530 \mu \mathrm{m})$ is etched by DRIE, the process wafer is separated from the handle wafer with acetone. After wafer cleaning, the exposed oxide layer is directionally dry-etched from the back side. Finally, the remaining masking LTO and exposed buried oxide layer is directionally etched from the front side.

\section{O. Device Characterization}

The two-dimensional (2D) MEMS scanner is actuated by electrostatic vertical combdrive actuators [33]. Electrostatic actuation in each direction is provided by two sets of vertical comb actuators that generate a large force to produce sizable deflection angles. The scanning electron micrograph (SEM) of the scanner is shown in Fig. 18. There are 4 actuation voltages $\left(\mathrm{V}_{1}, \mathrm{~V}_{2}, \mathrm{~V}_{3}\right.$, and $\left.\mathrm{V}_{4}\right)$ that power the device. The parameters of the scanner are characterized for quality control purposes prior to use in the miniature dual axes confocal microscope. First, the flatness of the mirror is measured with an interferometric surface profiler to identify micro-mirrors that have a peak-to-valley surface deformation $<0.1 \mu \mathrm{m}$. The scanner is metalized with $10 \mathrm{~nm}$ thick aluminum (reflectivity $=67 \%$ at $785 \mathrm{~nm}$ wavelength) to increase reflectivity.

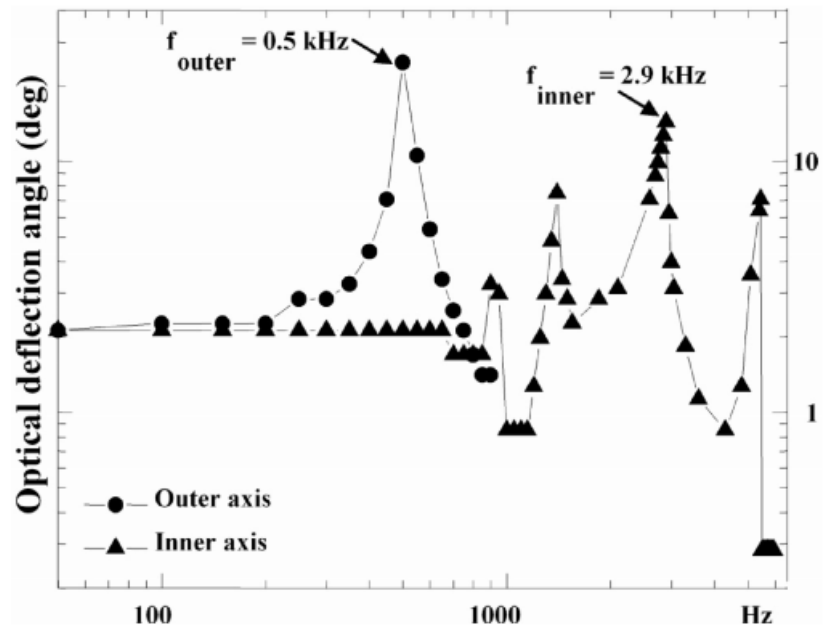

Fig. 19. Frequency response of MEMS mirror shows resonant peaks at $0.5 \mathrm{kHz}$ (outer axis) and $2.9 \mathrm{kHz}$ (inner axis) to achieve real time operation.

The radius of curvature of the mirror is greater than $60 \mathrm{~cm}$ with an average surface roughness of $7 \mathrm{~nm}$. Static optical deflections of $\pm 1.5 \mathrm{deg}$ at 180 volts and \pm 4.25 deg at 150 volts are achieved for the outer and inner axis, respectively. The resonant frequencies are 0.5 
$\mathrm{kHz}$ with \pm 10 deg optical angle at resonance for the outer axis and $2.9 \mathrm{kHz}$ with $\pm 17 \mathrm{deg}$ optical angle at resonance for the inner axis. The frequency response of the device is shown in Fig. 19. The parametric resonances can sometimes be observed in the inner axis near frequencies of $2 f_{0} / n$, where $n$ is an integer $\geq 1$ [34]. This phenomenon is caused by the nonlinear response of the torsional combdrives, which leads to sub-harmonic oscillations.

\section{Dual axes scanhead}

\section{P. Scanhead design}

The design and integration of the miniature dual axes scanhead is a very challenging part of the development of this novel imaging technique because of the small size required for compatibility with medical endoscopes. This process requires a package that allows for precise mounting of the following optical elements: 1) two fiber-coupled collimators, 2) 2D MEMS scanner, 3) parabolic focusing mirror, and 4) hemispherical index-matching solidimmersion-lens (SIL) [35]. The basic design of the miniature scanhead is shown in Fig. 20. Two collimated beams are focused at an inclination angle $\theta$ to the z-axis by a parabolic mirror with a maximum cone half-angle $a$ to an overlapping focal volume below the tissue surface after being deflected by the 2D MEMS scanner. The flat side of the SIL is placed against the tissue, and the curve surface accommodates the incident beams at normal incidence to minimize aberrations. The parabolic mirror is fabricated using a replicated molding process that provides a surface profile and smoothness needed for diffractionlimited focusing of the two collimated beams. Once the beams are aligned parallel to each other, the parabolic mirror then provides a "self-aligning" property that forces the focused beams to intersect at a common focal point below the tissue surface. Focusing is performed primarily by the parabolic mirror which is a non-refractive optical element with an NA of 0.12. This feature allows for the optical design to be achromatic. That is, light over a broad spectral regime can be focused to the same point below the tissue surface simultaneously, allowing for future multi-spectral imaging to be performed.

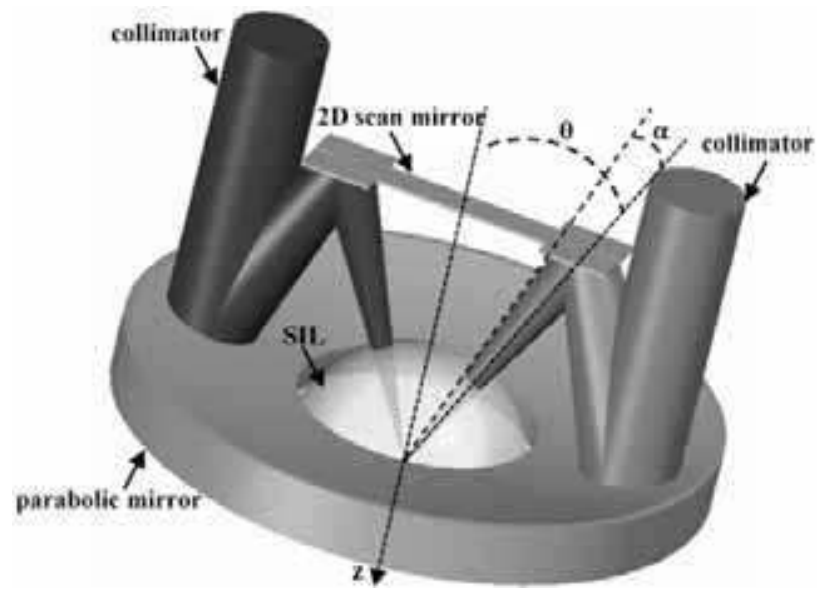

Fig. 20. Miniature dual axes scanhead. Two collimated beams that are focused by a parabolic mirror at angle $\theta$ to the $z$-axis for en face scanning by the 2D MEMS mirror. The solidimmersion lens (SIL) minimizes aberrations to the incident beams. 


\section{Q. Scanhead alignment and packaging}

Alignment of the two beams in this configuration is a key step to maximizing imaging performance. This step is accomplished by locating the two fiber-pigtailed collimators in a pair of v-grooves that are precision machined into the housing, as shown in Fig. 21a [35]. An accuracy of $0.05 \mathrm{deg}$ can be achieved in aligning the two beams parallel to one another using the v-grooves with pre-assembled fiber collimators. Additional precision in alignment can be attained with use of Risley prisms (optical wedges) introduced into the light paths to provide fine steering of the collimated beams to bring the system into final alignment. These prisms are angled at $0.1 \mathrm{deg}$, and can be rotated to steer the collimated beam in an arbitrary direction over a maximum range of $\sim 0.05 \mathrm{deg}$. This feature maximizes the overlap of the two beams after they are focused by the parabolic mirror. Two wedges are used in each beam so that complete cancellation of the deflection by each can be achieved, if needed, to provide maximum flexibility.
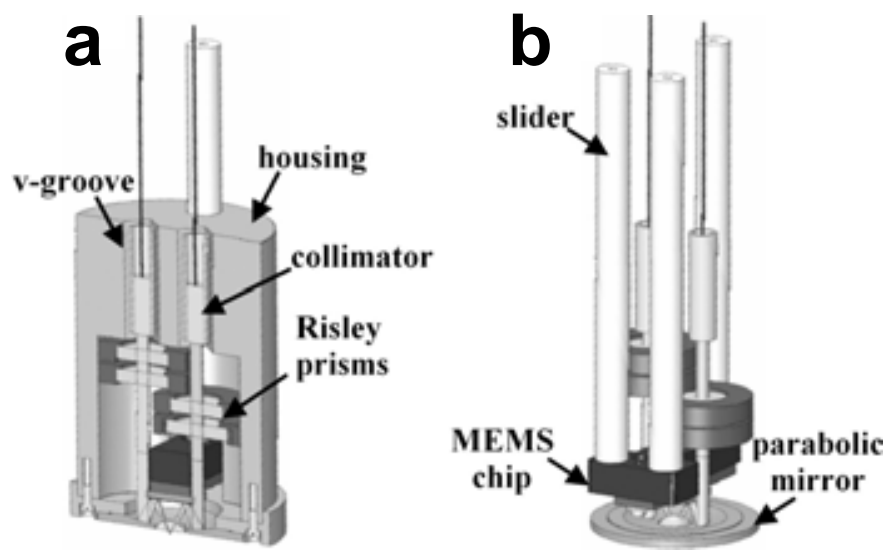

Fig. 21. Alignment and assembly of dual axes scanhead. a) Precision machined v-grooves and Risley prisms provide coarse and fine alignment, respectively, of the two beams. $b$ ) Axial (z-axis) displacement of the MEMS chip is made with a slider mechanism.

Axial (z-axis) displacement of the MEMS chip is performed with a computer-controlled piezoelectric actuator (Physik Instrumente $\mathrm{GmbH}$, P-783.ZL actuator, and E-662.LR controller) that moves a slider along 3 mechanical supports, shown in Fig. 21b. This feature adjusts the imaging depths to collect a stack of en face images to produce the 3D volume rendered images. The distal end of the slider has a mounting surface to attach the printed circuit board (PCB), which supports the MEMS chip, wire bonding surfaces, and soldering terminals.

A mixture of conductive epoxy (adhesive resin ECCOBOND Solder $56 \mathrm{C}$ and Catalyst 9, Emerson \& Cuming, Inc.) is used to attach aluminum-1\% silicon bonding wire (Semiconductor Packaging Materials, Inc.) from the bond pads on the MEMS die to that on the PCB. Electrical power is delivered to the mirror via wires that run through the middle of the housing, and are soldered onto the PCB terminals. The z-axis translational stage consists of a closed-loop piezoelectric linear actuator. Finally, the scanhead assembly is covered and sealed from the environment using UV-curing glue to prevent inward leakage of bodily fluids. 

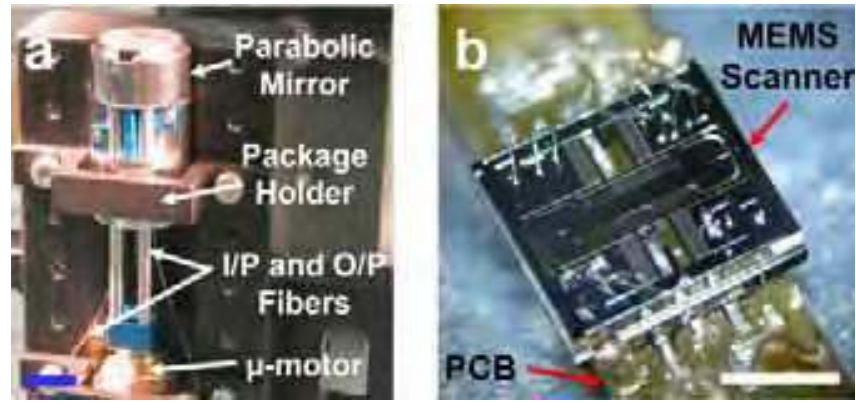

Fig. 22. a) Assembly of the dual axes confocal scanhead mounted on a V-block. b) Gimbaled 2D MEMS scanner wire bonded onto the PCB, scale bar $2 \mathrm{~mm}$.

Packaging of the $10 \mathrm{~mm}$ diameter scanhead mounted on a V-block stage is shown in Fig. 22a. A piezoelectric (micro) $\mu$-motor is used to perform vertical depth translation (z-axis). The MEMS scanner (die size is $3.2(\mathrm{w}) \times 2.9(\mathrm{~h}) \mathrm{mm}^{2}$ ) mounted on the PCB is shown in Fig. $22 b$.

\section{$R$. Instrument control and data acquisition}

Both the data acquisition and MEMS actuation systems are controlled using LabVIEWTM with Vision Acquisition software package and two National Instruments data acquisition (DAQ) boards (PXI-6711 and PXI-6115). The frequency and amplitude of the actuation signals control the frame rate and FOV of the MEMS scanner. There are 4 live and 1 ground wires that provide voltage to the device and are connected to the wirebond pads on the PCB via an ultrasonic wedge bonding technique.

For each 2D en face image, the MEMS scanner is resonantly driven 180 deg out of phase to maximize the linear region of the angular deflection $[14,29]$ around the outer axis $\left(\mathrm{V}_{1}\right.$ and $\mathrm{V}_{2}$ ) at $1.22 \mathrm{kHz}$ with a unipolar sine wave at a maximum of $70 \mathrm{~V}$, while rotation around the MEMS scanner inner axis $\left(\mathrm{V}_{3}\right.$ and $\left.\mathrm{V}_{4}\right)$ is driven 180 deg out of phase in the DC mode $(5 \mathrm{~Hz})$ with a unipolar sawtooth waveform at a maximum of $200 \mathrm{~V}$ (AgilOptics, Inc). The unipolar sawtooth waveform is smoothed at the transition edges to mitigate higher frequency ringing from the inner axis. The step size and depth scan range of the piezoelectric actuator (vertical translation) can be adjusted to optimize the acquisition of the 3D datasets.

The PMT gain is synchronously adjusted to compensate for reduced light collected at increased tissue depths. Automated frame averaging and display can be performed to reduce noise and improve image quality during imaging. 2D en face images from the analog input channel are acquired and displayed in real time to enable continuous monitoring or visualization of the sample. All images are acquired in 16-bit data format. 3D volumetic data can be rendered by post-processing using Amira ${ }^{\circledR}$ software (Visage Imaging, Inc).

\section{Prototype systems}

\section{S. Handheld instrument}

We first developed a $10 \mathrm{~mm}$ diameter handheld instrument, schematic shown in Fig. 23a $[14,29]$. A semiconductor laser delivers $25 \mathrm{~mW}$ of light $(\lambda=785 \mathrm{~nm})$ into a single mode fiber (SMF, Fibercore Limited, SM750). The fiber terminates in a $1.8 \mathrm{~mm}$ diameter gradient index (GRIN) collimator (GRINTECH, GmbH). The output beam diameter $\left(1 / \mathrm{e}^{2}\right)$ from the 
collimator is $0.9 \mathrm{~mm}$. The half angle $\theta$ between the input (illumination) and output (collection) beams is $24.3^{\circ}$. The input beam is focused by an aluminum coated parabolic mirror (PM) with the focal length of $4.6 \mathrm{~mm}$ (Anteryon BV) and reflects off the first mirror surface of the MEMS scanner. The center-to-center distance between the two collimators is $3.7 \mathrm{~mm}$. The focused beam continues through a fused silica SIL (hemispheric lens) until it reaches the focal plane below the tissue surface. The SIL has a refractive index (1.47) that is similar to that of the tissue and this material was chosen for index matching. The beams enter the air-silica interface at normal incidence to minimize aberrations as the focal point is scanned. Scattered light from the overlapping focal volume is collected through the optical window provided by the SIL and reflected off the MEMS mirror to the opposite surface of the parabolic mirror. The collected light is then focused onto the output fiber collimator for delivery to the PMT.
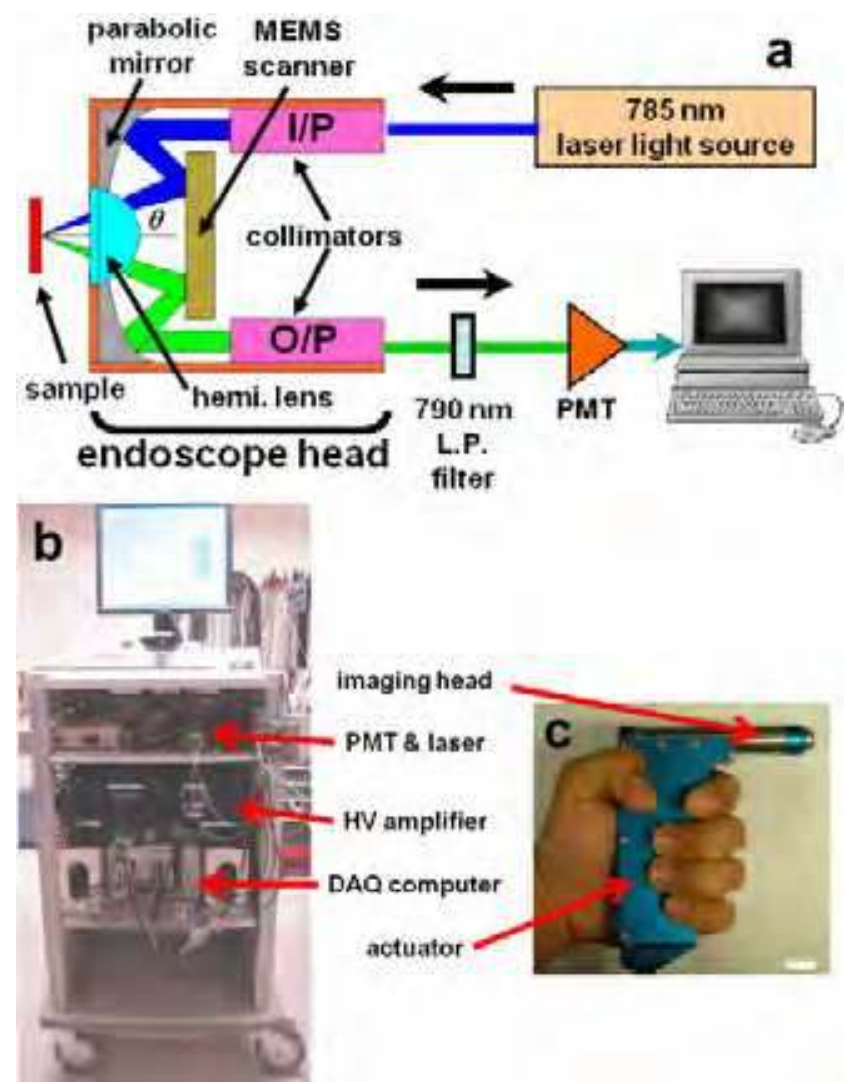

Fig. 23. Handheld prototype system. a) Schematic of complete instrument. b) Portable system demonstrated. c) Packaged handheld (10 mm diameter) dual axes confocal microscope with piezoelectric actuator in the handle, scale bar $10 \mathrm{~mm}$.

As the MEMS mirror raster scans the overlapping beams, the $2 \mathrm{D}$ en face image is continuously displayed on a computer monitor using a frame grabber and image acquisition software. Intensity mapping of each 2D en face image is performed by reading the MEMS 
scanner driving voltages and estimating focal beam trajectory. A 3D volumetric stack is created with post-processing and rendering a series of $2 \mathrm{D}$ en face images. Each 3D volumetric image is obtained by translating the MEMS scanner with the piezoelectric micromotor in the $z$-direction under computer-control. Imaging can be performed in reflectance or fluorescence mode by inserting a $790 \mathrm{~nm}$ long pass optical filter (LP-02-785RU-25, Semrock, Inc.) in the collection path for the latter case. The maximum output laser power on the sample is $2 \mathrm{~mW}$. A photograph of a fully-packaged miniature dual axes confocal microscope is shown in Fig. 23c.

\section{T. Endoscope-compatible instrument}

We scale down the basic design of the $10 \mathrm{~mm}$ diameter handheld instrument to develop the $5.5 \mathrm{~mm}$ endoscope-compatible version, shown in Fig. 24 [36]. This prototype uses the same replicated parabolic focusing and MEMS mirrors as that employed in the larger prototype. A pair of smaller $(1 \mathrm{~mm})$ diameter fiber-coupled GRIN collimator lenses is used in the smaller version. Alignment is provided by a pair of $1 \mathrm{~mm}$ diameter rotating wedges (Risley prisms), which are inserted into the path of one of the collimated beams. The collimators and Risley prisms are both located by precision wire-EDM machined v-grooves and epoxied into place with UV curing glue. As with the larger prototype, the combined precision of the v-grooves and the pointing accuracy of the pre-assembled fiber collimators allow for the collimated beams to become parallel to each other to within $\sim 0.05 \mathrm{deg}$ accuracy. The alignment wedges have a small $(0.1 \mathrm{deg})$ angle, which allows for steering of a collimated beam over a range of about $0.05 \mathrm{deg}$ in any direction as each wedge is rotated.

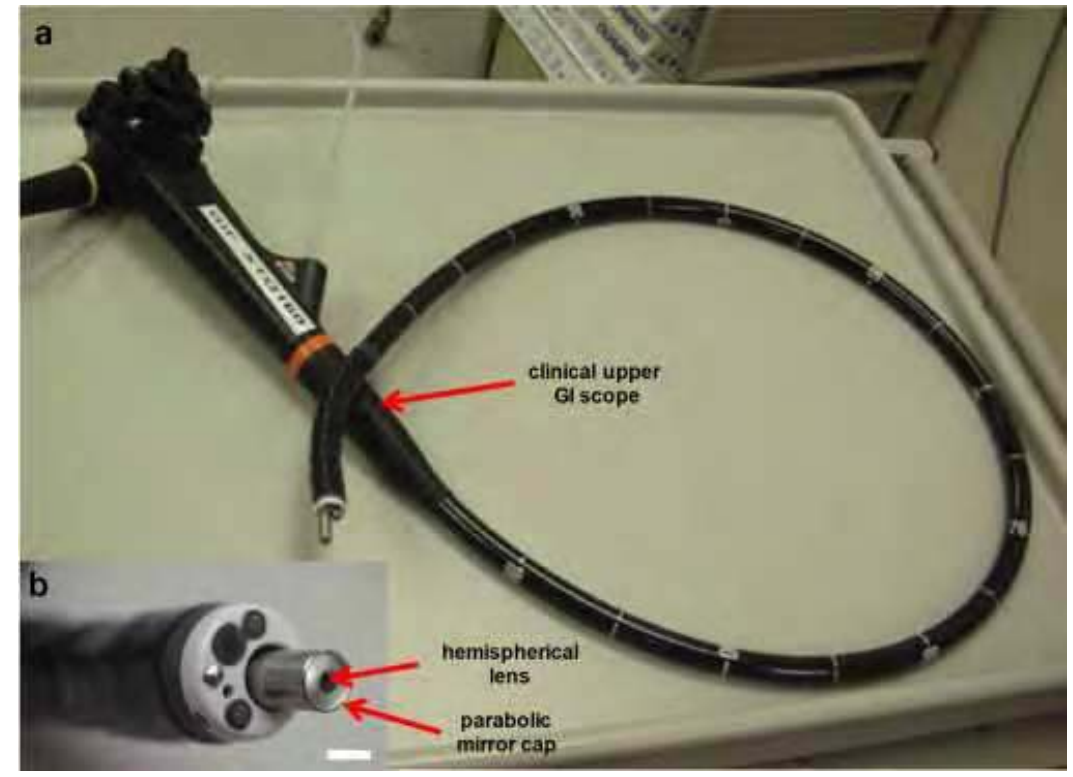

Fig. 24. Endoscope-compatible dual axes confocal microscope. a) Microscope passes through the instrument channel of an Olympus XTQ 160 therapeutic upper endoscope that has a 6 mm diameter instrument channel. b) Distal end of endoscope shows the protruding dual axes microendoscope. 
This smaller package design also accommodates a slider mechanism, which is used for axial (z-axis) scanning of the MEMS chip to provide a variable imaging depths within the tissue and for generating 3D volumetric images. This smaller slider mechanism comprises a single rod, which moves within a precision hole drilled through the housing. The MEMS chip is mounted by an adhesive to a PCB, which is in-turn mounted onto the slider. The PCB provides bondpads to accommodate wire bonding to the MEMS chip and also to provide soldering terminals for the external control wires that power the scan mirror. In Fig. 24a, the endoscope-compatible dual axes confocal microscope is shown inserted through the $6 \mathrm{~mm}$ diameter instrument channel of a therapeutic upper endoscope (Olympus GIF XTQ160). A magnified view of the distal tip is shown in Fig. 24b.

\section{Imaging results}

\section{U. Reflectance imaging}

Instrument characterization is performed in reflectance mode by imaging a chrome surface of a standard (USAF) resolution target. It is also used as a sample to measure the image resolution and FOV. The transverse resolution was measured by the knife-edge method, defined by $10 \%$ to $90 \%$ of maximum intensity points, and found to be $5 \mu \mathrm{m}$ [36]. The axial resolution, defined by FWHM, is measured by translating a plane mirror in the $z$-direction and was found to be $7 \mu \mathrm{m}$.

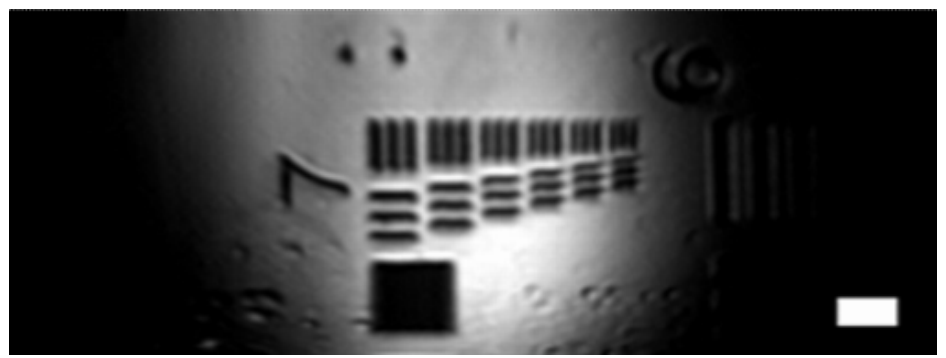

Fig. 25. Reflectance image of standard (USAF) resolution target collected with handheld dual axes confocal microscope, scale bar $20 \mu \mathrm{m}$.

Fig. 25 shows a reflectance image collected with the handheld confocal microscope that reveals clear visualization of group 7 of the USAF resolution target. The measured values are slightly larger than the theoretical resolutions of $4.5 \mu \mathrm{m}$ for the transverse dimensions, and $6.0 \mu \mathrm{m}$ for the axial dimension. This is mainly due to the decrease in effective NA of the imaging system from the truncation of both input and output collimated beams by the width dimension of the MEMS scanner die. All acquired images are captured at 5 frames/second with the largest FOV of $800 \times 450 \mu \mathrm{m}^{2}\left(900 \times 506\right.$ pixels $\left.^{2}\right)$. This FOV is much larger than that most other miniature confocal instruments, and is achieved with use of post-objective scanning.

\section{$V$. Ex vivo fluorescence imaging}

\section{Handheld dual axes confocal instrument}

The 3D fluorescence imaging capability of the handheld dual axes confocal instrument is shown in Fig. 26. Excised tissue specimens of normal and dysplastic colonic mucosa are 
soaked in $0.5 \mathrm{mg}$ of LI-COR IRDye ${ }^{\circledR} 800$ CW NHS Ester (LI-COR Biosciences, Inc) diluted in $10 \mathrm{ml}$ of phosphate-buffered saline (PBS) at neutral $\mathrm{pH}$ for 5 minutes and then rinsed with water to remove excess dye. After imaging, the specimens are fixed in $10 \%$ buffered formalin, cut into $5 \mu \mathrm{m}$ sections, and processed for histology with hematoxylin and eosin $(\mathrm{H} \& \mathrm{E})$. All ex vivo images are obtained from freshly excised human tissues (obtained with informed consent at the VA Palo Alto Health Care System).
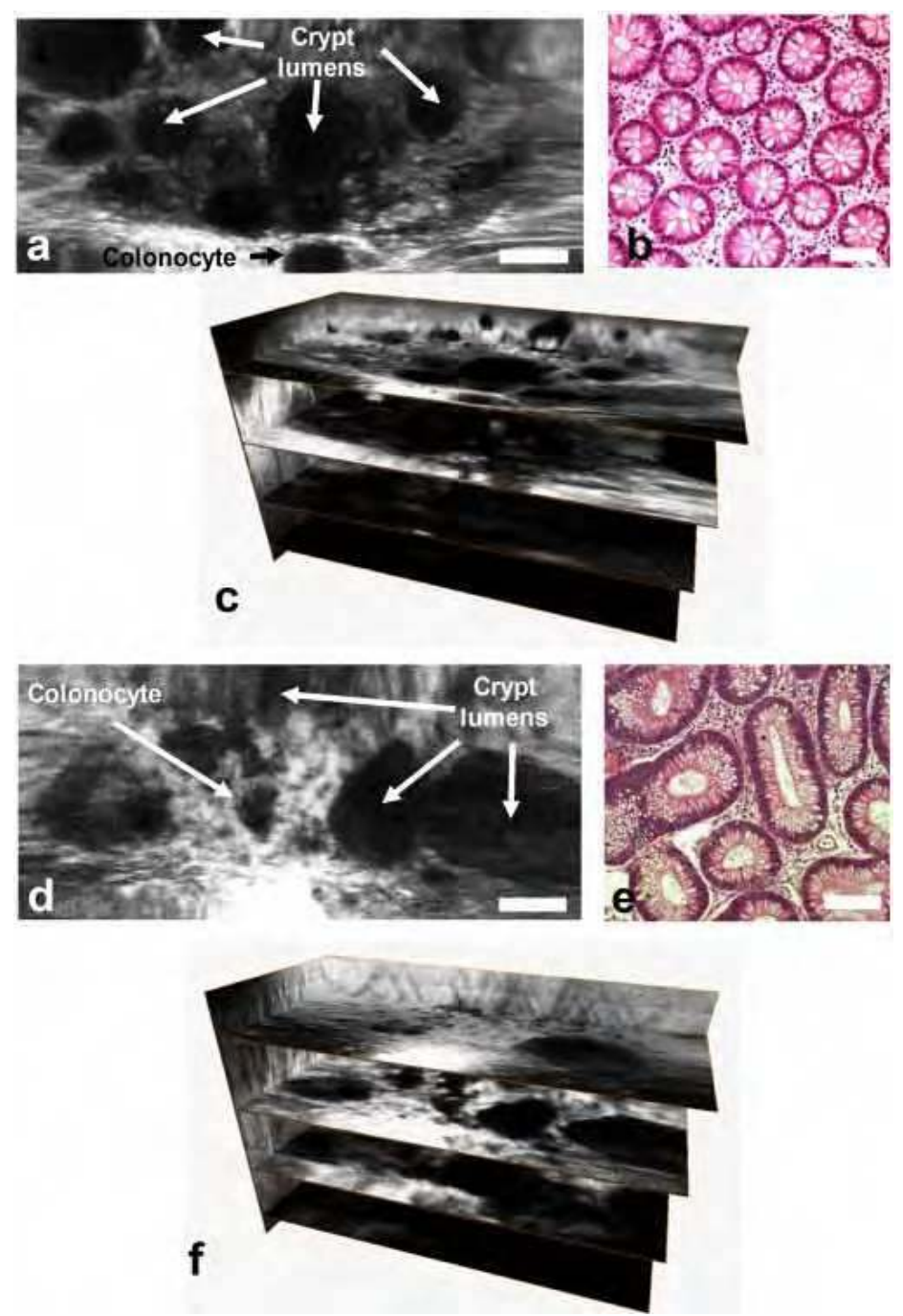

Fig. 26. Ex vivo images. En face dual axes confocal images of a) normal, d) dysplastic colonic mucosa. Corresponding histology (H\&E) of b) normal and e) dysplasia. 3D volumetric images of c) normal and f) dysplasia, scale bar $100 \mu \mathrm{m}$. 
Fig 26a, b, and c and Fig 26d, e, and f show the en face image, histology (H\&E), and 3D volumetric images of normal and adenomatous (dysplastic) colonic mucosa, respectively, scale bar $100 \mu \mathrm{m}[36]$. Features of colonic crypts, including colonocytes and crypt lumens, are clearly resolved. Fig. 26c and 24f show three extracted en face planes at 50, 170, and 230 $\mu \mathrm{m}$ below the tissue surface. The gain is increased with depth to compensate for the lower signal levels.

\section{Endoscope-compatible dual axes confocal instrument}

A 2D en face fluorescence image of normal colonic mucosa collected ex vivo with the $5.5 \mathrm{~mm}$ diameter endoscope-compatible dual axes confocal prototype is shown in Fig. 27, scale bar $100 \mu \mathrm{m}$. ICG was topically applied to enhance contrast, and the pseudocolor image shows dye enhancement in the lamina propria surrounding the circular shaped crypts.

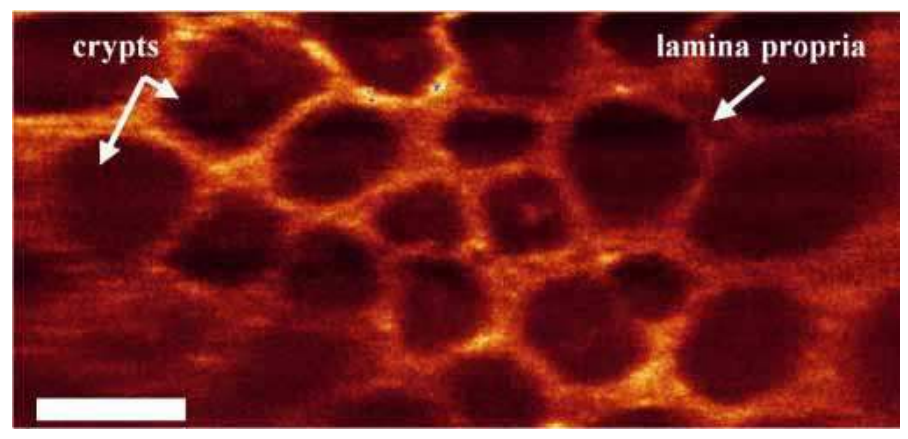

Fig. 27. En face fluorescence image of normal colonic mucosa collected with endoscopecompatible dual axes confocal prototype ex vivo using topically applied ICG to enhance contrast shows regular crypt pattern, scale bar $100 \mu \mathrm{m}$.

W. In vivo fluorescence imaging

In vivo imaging with the handheld dual axes confocal microscope has also been demonstrated. A mouse was anesthetized, and $10 \mathrm{mg}$ of indocyanine green (Sigma-Aldrich, Corp) diluted in $10 \mathrm{ml}$ of PBS was injected into the retro-orbital plexus of the mouse. Imaging was performed by resting the mouse on a translational stage and placing its ear intact on the SIL window of the microscope. Fig. 28a shows an in vivo image of blood vessels en face with a maximum intensity projection. Fig. $28 \mathrm{~b}$ shows a $3 \mathrm{D}$ volumetric rendering of the image stack obtained by scanning from the surface to $150 \mu \mathrm{m}$ deep into the intact ear. The images were collected in $3 \mu \mathrm{m}$ intervals along the z-axis by using the piezoelectric actuator. All images were taken at $5 \mathrm{~Hz}$ with 5 frames averaging ( 1 second per image). The full 3D volume rendered image was acquired in 50 seconds, scale bar $100 \mu \mathrm{m}$.

In addition, in vivo images of human skin collected with the handheld dual axes confocal microscope are shown in Fig. 29. A sequence of approximately 300 individual en face images of human skin were collected at a fixed depth of $60 \mu \mathrm{m}$ below the tissue surface (stratum corneum) with a speed of $5 \mathrm{~Hz} \mu \mathrm{m}$ [36]. Topically applied indocyanine green was used for contrast. Image stitching or mosaicing was performed to enlarge the FOV and to increase the signal-to-noise ratio in real time with custom mosaicing software, shown in Fig. 29a. The 

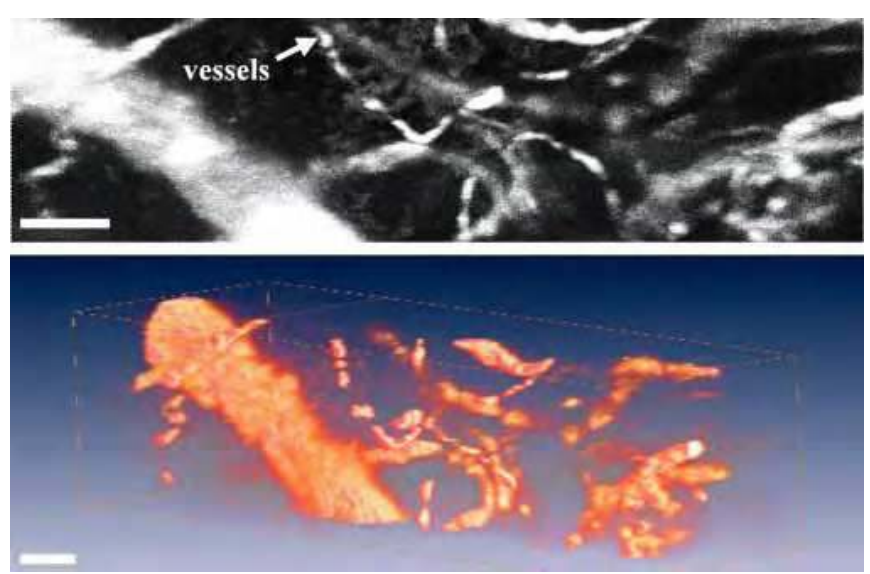

Fig. 28. a) A maximum intensity projected in vivo image of blood vessels in an intact mouse ear collected with handheld prototype. b) A 3D volume rendered image of blood vessels, scale bar $100 \mu \mathrm{m}$.
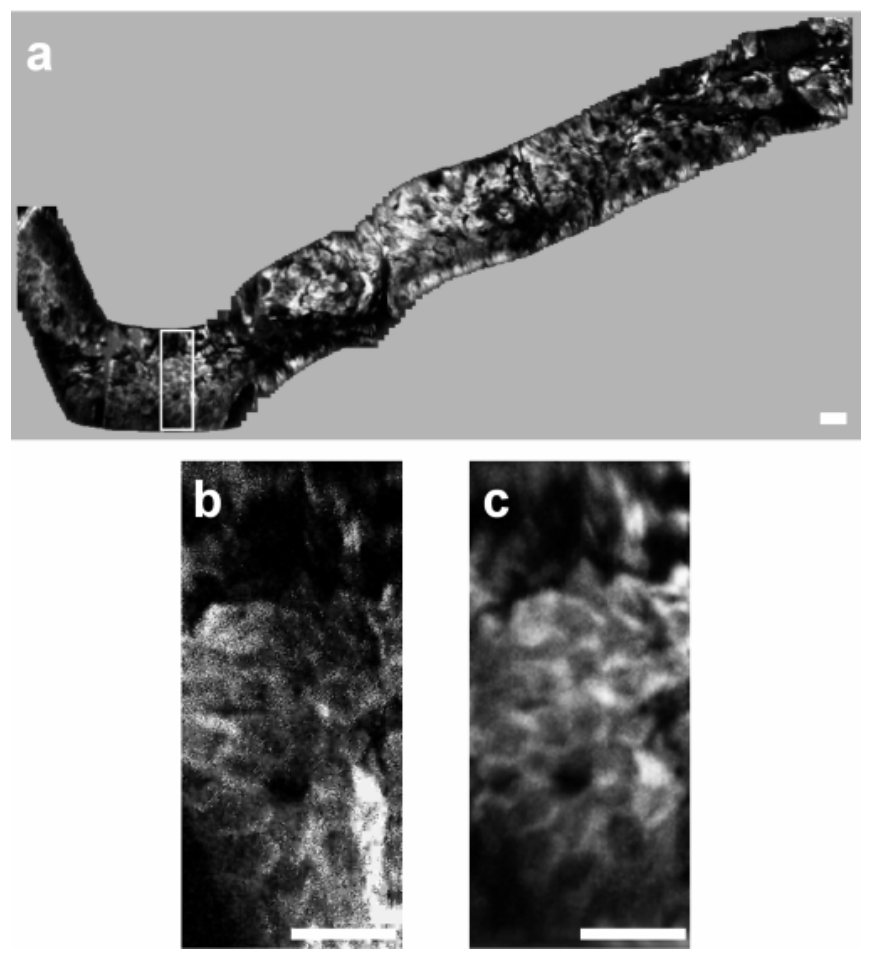

Fig. 29. a) Image mosaic of human skin acquired in vivo at a depth of $60 \mu \mathrm{m}$ composed of roughly 300 images. The white box shows the corresponding location of individual images, b) A single input image; c) the corresponding area of the mosaic with improved signal-tonoise ratio, scale bars $50 \mu \mathrm{m}$. 
white rectangle box in Fig. 29a represents an individual en face image $\left(100 \times 400 \mu \mathrm{m}^{2}\right)$ obtained with the dual axes confocal microscope. The images were mosaiced by first correcting the image borders for scanning distortions. Then, each new image was registered and blended before proceeding to the next. Fig. $29 \mathrm{~b}$ and $29 \mathrm{c}$ demonstrate how image mosaicing can increase the signal to noise ratio and dramatically improve image quality by tuning the amount of image overlap. The maximum input frame rate that our computer acquisition can process with the real-time mosaicing algorithm is 15 frames/second.

\section{Conclusions and future directions}

In this chapter, we present the theory, design and implementation of a novel dual axes confocal microscope in both tabletop and miniature form factors. Separate illumination and collection of light using the region of overlap between the two beams (focal volume) provide a number of advantages for purposes of miniaturization and in vivo imaging. The instruments were developed with $785 \mathrm{~nm}$ illumination to take advantage of the "optical window" in tissue where the high dynamic range and deep tissue penetration of this novel architecture can be demonstrated. This instrument is able to achieve sub-cellular resolution $(\sim 5 \mu \mathrm{m})$, sufficient for in vivo histopathological evaluation. Performance of the dual axes confocal microscope is demonstrated by collecting both en face images in real time and 3D volumetric images with post-processing at a maximum interrogating depth of $300 \mu \mathrm{m}$ for both ex vivo and in vivo samples. Furthermore, we used this instrument as a test bed to further scale down the dimensions of this architecture to a $5.5 \mathrm{~mm}$ diameter package for endoscope compatibility. The size of the instrument has been reduced with a more compact aligning mechanism.

We have demonstrated a tissue penetration depth with the dual axes confocal microscope that is unmatched by any other endoscope-compatible instrument. From our in vivo experiments, fluorescence images can be collected up to a depth of $300 \mu \mathrm{m}$, limited by the maximum travel of the piezoelectric actuator. Greater depths have been achieved with our tabletop instruments $(>500 \mu \mathrm{m})$. These results demonstrate the large working distance and high dynamic range of the dual axes confocal architecture to enable deep subsurface tissue imaging. Further improvements in performance can be achieved by increasing light throughput. The relatively low output power of $2 \mathrm{~mW}$ can be significantly increased with use of either silver or gold coatings as the reflective surfaces of the MEMS scanner and parabolic mirror, rather than aluminum. In addition, a higher power fiber-coupled laser source can be used.

Future development of dual axes confocal architecture will focus on achieving the theoretical levels of performance in a miniature instrument package. In addition, repeatability and reliability will be addressed. We will take advantage of the high dynamic range of the system by developing new z-axis actuators that rapidly scan the focal volume perpendicular to the tissue surface to achieve deep penetration in vertical cross-sections. This orientation provides a powerful view for studying the epithelium and presents a comprehensive picture of the biological differentiation patterns in this thin layer of metabolically active tissue. The epithelium forms the inner lining of all hollow organs, and is accessible by medical endoscopes. In addition, we will extend this approach to multispectral imaging capabilities by developing achromatic optics using the same basic optical 
design. Finally, smaller form factors will be developed to achieve compatibility with standard medical endoscopes.

As this novel approach matures, we will be able to use this high resolution imaging instrument to perform clinical investigation in human subjects and longitudinal studies in small animal models. Molecular specificity can be achieved by combining this microendoscope with use of affinity probes that bind to over expressed cell surface targets. This integrated imaging methodology will provide the ability to visualize molecular features of tissue micro-structures in the vertical plane with sub-mucosal axial depths. This powerful capability has tremendous potential to unravel previously unknown molecular mechanisms about important disease processes, such as cancer and inflammation.

\section{Acknowledements}

We thank Christopher H Contag, Shai Friedland, Gordon S Kino, Jonathon TC Liu, Michael J Mandella, Hyejun Ra, Roy M Soetikno, Olav Solgaard, and Larry K Wong for their technical support.

\section{References}

[1] R. S. Cotran, V. Kumar, and T. Collins, "Robbins Pathologic Basis of Disease, 6th ed.," Philadelphia: W. B. Saunders Company, 1999, pp. 781-87.

[2] J. Pawley, Handbook of biological confocal microscopy, 3rd ed. New York: Plenum Press, 1996.

[3] T. R. C. a. G. S. Kino, "Confocal Scanning Optical Microscopy and Related Imaigng Systems, Academic Press, Boston," 1996.

[4] E. Laemmel, M. Genet, G. Le Goualher, A. Perchant, J. F. Le Gargasson, and E. Vicaut, "Fibered confocal fluorescence microscopy (Cell-viZio) facilitates extended imaging in the field of microcirculation. A comparison with intravital microscopy," Journal of Vascular Research, vol. 41, pp. 400-411, Sep-Oct 2004.

[5] K. Carlson, M. Chidley, K.-B. Sung, M. Descour, A. Gillenwater, M. Follen, and R. Richards-Kortum, "In vivo fiber-optic confocal reflectance microscope with an injection-molded miniature objective lens," Applied Optics, vol. 44, pp. 1792-1796, April 1, 2005.

[6] N. Thekkek and R. Richards-Kortum, "Optical imaging for cervical cancer detection: solutions for a continuing global problem," Nat Rev Cancer, vol. 8, pp. 725-731, 2008.

[7] P. M. Delaney, M. R. Harris, and R. G. King, "Fiber-optic laser scanning confocal microscope suitable for fluorescence imaging," Applied Optics, vol. 33, pp. 573-577, February 1, 1994.

[8] T. D. Wang, S. Friedland, P. Sahbaie, R. Soetikno, P.-L. Hsiung, J. T. C. Liu, J. M. Crawford, and C. H. Contag, "Functional Imaging of Colonic Mucosa With a Fibered Confocal Microscope for Real-Time In Vivo Pathology," Clinical Gastroenterology and Hepatology, vol. 5, pp. 1300-1305, 2007. 
[9] D. L. Dickensheets and G. S. Kino, "A scanned optical fiber confocal microscope," in SPIE Symposium on Electronic Imaging Science \& Technology, San Jose, CA, 1994, pp. 39-47.

[10] D. L. Dickensheets and G. S. Kino, "Silicon-micromachined scanning confocal optical microscope," Journal of Microelectromechanical Systems, vol. 7, pp. 38-47, March, 1998.

[11] W. Piyawattanametha, H. Toshiyoshi, J. LaCosse, and M. C. Wu, "Surfacemicromachined confocal scanning optical microscope," Conference on Lasers and Electro-Optics (CLEO 2000), May 7-12 2000, San Francisco, CA, USA, pp. 447-448, 2000.

[12] K. Murakami, A. Murata, T. Suga, H. Kitagawa, Y. Kamiya, M. Kubo, K. Matsumoto, H. Miyajima, and M. Katashiro, "A miniature confocal optical microscope with MEMS gimbal scanner.," in IEEE International Solid-State Sensors and Actuators Conference, June 8-12, 2003, Boston, MA, USA, 2003, pp. 587-590, vol.1.

[13] W. Piyawattanametha, L. Fan, S. Hsu, M. Fujino, M. C. Wu, P. R. Herz, A. D. Aguirre, Y. Chen, and J. G. Fujimoto, "Two-dimensional endoscopic MEMS scanner for high resolution optical coherence tomography.," in on Lasers and Electro-Optics (CLEO), May 16-21, 2004, San Francisco, CA, USA, 2004, pp. 3, vol.1.

[14] W. Piyawattanametha, P. R. Patterson, D. Hah, H. Toshiyoshi, and M. C. Wu, "Surfaceand bulk- micromachined two-dimensional scanner driven by angular vertical comb actuators.," Journal of Microelectromechanical Systems, vol. 14, pp. 1329-1338, 2005.

[15] L. Thiberville, S. Moreno-Swirc, T. Vercauteren, E. Peltier, C. Cave, and G. Bourg Heckly, "In Vivo Imaging of the Bronchial Wall Microstructure Using Fibered Confocal Fluorescence Microscopy," Am. J. Respir. Crit. Care Med., vol. 175, pp. 2231, January 1, 20072007.

[16] T. D. Wang, M. J. Mandella, C. H. Contag, and G. S. Kino, "Dual-axis confocal microscope for high-resolution in vivo imaging," Optics Letters, vol. 28, pp. 414-416, March 15, 2003.

[17] T. D. Wang, C. H. Contag, M. J. Mandella, N. Y. Chan, and G. S. Kino, "Dual-axes confocal microscopy with post-objective scanning and low-coherence heterodyne detection," Optics Letters, vol. 28, pp. 1915-1917, October 15, 2003.

[18] M. Born and E. Wolf, Principles of Optics, 7th ed. ed.: Cambridge University Press, 1999.

[19] J. T. C. Liu, M. J. Mandella, S. Friedland, R. Soetikno, J. M. Crawford, C. H. Contag, G. S. Kino, and T. D. Wang, "Dual-axes confocal reflectance microscope for distinguishing colonic neoplasia," Journal of Biomedical Optics, vol. 11, pp. -, SEPOCT 2006.

[20] L. K. Wong, M. J. Mandella, G. S. Kino, and T. D. Wang, "Improved rejection of multiply scattered photons in confocal microscopy using dual-axes architecture," Optics Letters, vol. 32, pp. 1674-1676, June 15, 2007.

[21] C. Henyey, Diffuse radiation in the Galaxy, 2000. 
[22] K. T. Mehta and H. S. Shah, "Correlating Parameters Of The Henyey-Greenstein Phase Function Equation With Size And Refractive-Index Of Colorants," Applied opticsOT, vol. 24, pp. 892-896, 1985.

[23] K. Pavlos, "Scattering, Absorption, and Emission of Light by Small Particles," Bulletin of the American Meteorological Society, vol. 84, p. 494, 2003.

[24] M. K. S. A. Prahl, S. L. Jacques, A. J. Welch, "Dosimetry of Laser Radiation in Medicine and Biology," Proc. SPIE, vol. 5, pp. 102-111 1989.

[25] T. D. Wang, C. H. Contag, M. J. Mandella, N. Y. Chan, and G. S. Kino, "Confocal fluorescence microscope with dual-axis architecture and biaxial postobjective scanning," J Biomed Opt, vol. 9, pp. 735-42, Jul-Aug 2004.

[26] T. D. Wang, C. H. Contag, M. J. Mandella, N. Y. Chan, and G. S. Kino, "Confocal fluorescence microscope with dual-axis architecture and biaxial postobjective scanning," Journal of Biomedical Optics, vol. 9, pp. 735-742, JUL-AUG 2004.

[27] J. T. C. Liu, M. Mandella, J. Crawford, C. Contag, T. Wang, and G. Kino, "Efficient rejection of scattered light enables deep optical sectioning in turbid media with low-numerical-aperture optics in a dual-axis confocal architecture," Journal of Biomedical Optics, vol. 13, p. 34020, 2008.

[28] D. L. Dickensheets and G. S. Kino, "Micromachined scanning confocal optical microscope," Optics Letters, vol. 21, pp. 764-766, May 15, 1996.

[29] W. Piyawattanametha, R. P. J. Barretto, T. H. Ko, B. A. Flusberg, E. D. Cocker, H. J. Ra, D. S. Lee, O. Solgaard, and M. J. Schnitzer, "Fast-scanning two-photon fluorescence imaging based on a microelectromechanical systems two-dimensional scanning mirror," Optics Letters, vol. 31, pp. 2018-2020, July 1, 2006.

[30] S. P. Timoshenko and J. N. Goodier, Theory of Elasticity, 3rd ed. New York: McGrawHill, 1970.

[31] W. Piyawattanametha, P. R. Patterson, D. Hah, H. Toshiyoshi, and M. C. Wu, "Surfaceand bulk-micromachined two-dimensional scanner driven by angular vertical comb actuators," Journal of Microelectromechanical Systems, vol. 14, pp. 1329-1338, DEC 2005.

[32] H. Ra, Y. Taguchi, D. Lee, W. Piyawattanametha, and O. Solgaard, "Two-dimensional MEMS scanner for dual-axes confocal in vivo microscopy," Proceedings of the 19th IEEE International Conference on Micro Electro Mechanical Systems (MEMS), Istanbul, Turkey, vol. 2006, pp. 862-865, 2006.

[33] H. Ra, W. Piyawattanametha, Y. Taguchi, D. Lee, M. J. Mandella, and O. Solgaard, "Two-dimensional MEMS scanner for dual-axes confocal microscopy," Journal of Microelectromechanical Systems, vol. 16, pp. 969-976, AUG 2007.

[34] C. Ataman and H. Urey, "Nonlinear Frequency Response of Comb-Driven Microscanners," Proceedings of SPIE - The International Society for Optical EngineeringMOEMS Display and Imaging Systems II; Jan 26-27 2004; San Jose, CA., United States, vol. 5348, pp. 166-174, 2004.

[35] J. T. C. Liu, M. J. Mandella, H. Ra, L. K. Wong, O. Solgaard, G. S. Kino, W. Piyawattanametha, C. H. Contag, and T. D. Wang, "Miniature near-infrared dualaxes confocal microscope utilizing a two-dimensional microelectromechanical systems scanner," Optics Letters, vol. 32, pp. 256-258, FEB 12007. 
[36] A. E. Siegman, M. W. Sasnett, and T. F. Johnston, "Choice of Clip Levels for Beam Width Measurements Using Knife-Edge Techniques," IEEE Journal of Quantum Electronics, vol. 27, pp. 1098-1104, April 1991. 


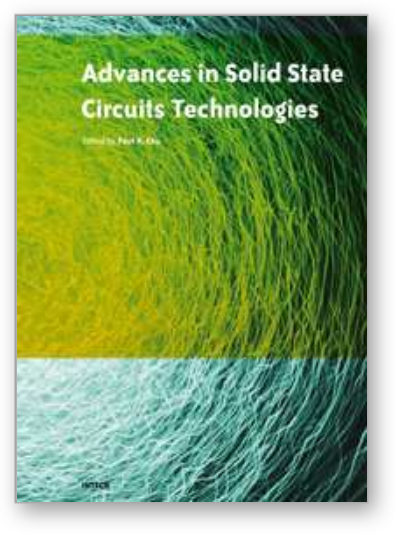

\author{
Advances in Solid State Circuit Technologies \\ Edited by Paul K Chu
}

ISBN 978-953-307-086-5

Hard cover, 446 pages

Publisher InTech

Published online 01, April, 2010

Published in print edition April, 2010

This book brings together contributions from experts in the fields to describe the current status of important topics in solid-state circuit technologies. It consists of 20 chapters which are grouped under the following categories: general information, circuits and devices, materials, and characterization techniques. These chapters have been written by renowned experts in the respective fields making this book valuable to the integrated circuits and materials science communities. It is intended for a diverse readership including electrical engineers and material scientists in the industry and academic institutions. Readers will be able to familiarize themselves with the latest technologies in the various fields.

\title{
How to reference
}

In order to correctly reference this scholarly work, feel free to copy and paste the following:

Wibool Piyawattanametha and Thomas D. Wang (2010). Miniature Dual Axes Confocal Microscope for Real Time In Vivo Imaging, Advances in Solid State Circuit Technologies, Paul K Chu (Ed.), ISBN: 978-953-307086-5, InTech, Available from: http://www.intechopen.com/books/advances-in-solid-state-circuittechnologies/miniature-dual-axes-confocal-microscope-for-real-time-in-vivo-imaging

\section{INTECH}

open science | open minds

\section{InTech Europe}

University Campus STeP Ri Slavka Krautzeka 83/A 51000 Rijeka, Croatia Phone: +385 (51) 770447

Fax: +385 (51) 686166 www.intechopen.com

\section{InTech China}

Unit 405, Office Block, Hotel Equatorial Shanghai No.65, Yan An Road (West), Shanghai, 200040, China 中国上海市延安西路65号上海国际贵都大饭店办公楼 405 单元 Phone: $+86-21-62489820$

Fax: $+86-21-62489821$ 
(C) 2010 The Author(s). Licensee IntechOpen. This chapter is distributed under the terms of the Creative Commons Attribution-NonCommercialShareAlike-3.0 License, which permits use, distribution and reproduction for non-commercial purposes, provided the original is properly cited and derivative works building on this content are distributed under the same license. 